\title{
De l'utilisation de la notion de risque dans le débat public sur les biotechnologies
}

\section{Barbara Bordogna Petriccione}

\section{(2) OpenEdition}

\section{Journals}

Édition électronique

URL : http://journals.openedition.org/ress/355

DOI : $10.4000 /$ ress.355

ISSN : 1663-4446

Éditeur

Librairie Droz

\section{Édition imprimée}

Date de publication : 1 novembre 2004

Pagination : 73-108

ISBN : 2-600-00980-9

ISSN : 0048-8046

\section{Référence électronique}

Barbara Bordogna Petriccione, « De l'utilisation de la notion de risque dans le débat public sur les biotechnologies », Revue européenne des sciences sociales [En ligne], XLII-130 | 2004, mis en ligne le 17 novembre 2009, consulté le 19 avril 2019. URL : http://journals.openedition.org/ress/355 ; DOI : 10.4000/ress.355 


\section{DE L'UTILISATION DE LA NOTION DE RISQUE DANS LE DÉBAT PUBLIC SUR LES BIOTECHNOLOGIES}

\section{INTRODUCTION}

Les impacts des biotechnologies appliquées au domaine agro-alimentaire suscitent de nombreuses préoccupations. Les incertitudes tant scientifiques qu'économiques, politiques, sociales ou éthiques au sujet des organismes génétiquement modifiés (OGM) sont caractéristiques de la controverse qui anime depuis plusieurs années l'espace public suisse, européen et plus récemment étatsuniens. La question des risques liés aux biotechnologies a occupé et occupe toujours une place centrale dans le débat public $^{1}$ et les acteurs sociaux - paysans, associations de consommateurs, groupements écologistes, mouvement altermondialiste - font très fréquemment recours à la notion de risque dans leur argumentation. L'utilisation de la notion de risque par les acteurs sociaux recouvre toutefois une réalité souvent très large alors qu'au contraire, les instances chargées d'encadrer le développement des biotechnologies donnent à ce terme une acception à la fois précise et limitée. Pour ces dernières, les risques ne peuvent être qu'environnementaux ou sanitaires, alors que pour les premiers, ils peuvent aussi être sociaux, économiques ou même éthiques.

Cet article se propose, dans un premier temps, d'examiner les raisons qui peuvent être à l'origine de cette utilisation très large de la notion de risque par les acteurs sociaux. L'accent sera mis sur la dimension stratégique de la notion de risque ainsi que sur la dynamique des acteurs qui en font usage. Dans un deuxième temps, la position des instances de régulation en ce qui concerne le risque sera analysée. Les critères permettant à ces instances de distinguer entre les risques qu'elles prennent en considération et les risques qui ne peuvent donner lieu à la prise de mesures d'évitement seront mis en évidence. Ces développements nous amènerons ensuite à considérer les justifications propres à chacune de ces acceptions de la notion de risque. Enfin, la question du principe de précaution sera examinée à l'aune des conclusions des précédentes sections.

\footnotetext{
Nous entendons par «débat public» des échanges de point de vue et/ou des confrontations sociales qui se déroulent de manière peu dirigée ou structurée par opposition aux dispositifs participatifs de type conférence de consensus ou publiforum, qui sont expressément mis en place pour générer un échange d'arguments et de points de vue destiné à évaluer divers choix technologiques. Cette définition est reprise de Joly P-B., Assouline G. (2001) «Assessing debate and participative technology assessment in Europe, Final report», Grenoble: INRA Economie et Sociologie rurales, Tey: QAP Decision, June 2001 - (ADAPTA PROJECT).
} 


\section{UTILISATION DE LA NOTION DE RISQUE PAR LES ACTEURS SOCIAUX}

\subsection{La diversité des risques}

Nous avons tenté de résumer de manière schématique dans un tableau (voir annexe 1) les principaux arguments qui apparaissent dans le débat public sur les OGM en termes de risques. Ils ont été classés en fonction du type d'acteur susceptible de les invoquer, sachant que les catégories d'acteurs ainsi introduites ne reflètent que très imparfaitement une réalité fort complexe où d'une part un individus peut être porteur de plusieurs «casquettes» d'acteur (par exemple consommateur, paysan et écologiste) et d'autre part un même risque peut être invoqué par différentes catégories d'acteurs.

Les risques sont également classés en fonction de deux paramètres: (1) le type de dommage susceptible de se réaliser et (2) la méthode par laquelle le risque est identifié et validé. Ces paramètres nous serviront par la suite à distinguer les risques pris en compte par les instances en charge d'encadrer le développement des biotechnologies (risques reconnus) de ceux qui ne le sont pas (risques non reconnus); leur teneur sera donc explicitée plus avant.

\subsection{Le risque: une exigence implicite de mise en œuvre de mesures d'évitement}

A la différence d'autres manières de donner forme à un argument, le recours à la notion de risque permet de conférer une dimension particulière à un discours. Le premier élément qu'il convient de mentionner porte sur la valeur normative plus ou moins importante qui est associée aux différentes manières de formuler un argument. Dans la perspective d'influencer le cours du développement des biotechnologies, quel poids pourrait en effet revêtir une argumentation développée en termes d'impacts, de questions, ou encore de dimensions? Un discours faisant état de «la question du maintien d'une agriculture biologique totalement exempte d'OGM grâce à l'application d'un moratoire» n'a évidemment pas la même force de conviction que celui qui invoquerait «le risque de ne plus pouvoir disposer de produit biologiques si un moratoire n'est pas appliqué ». En effet, le choix de ces termes n'est nullement anodin puisque certains d'entre eux permettent de qualifier un argument de manière normative, que ce soit positivement ou négativement, alors que d'autres restent relativement neutres. Le schéma cidessous permet de visualiser la connotation de quelques uns des termes les plus fréquemment utilisés dans le débat sur les OGM; le risque étant probablement l'un des termes connotés le plus négativement alors qu'à l'inverse, l'opportunité apparaît parmi les plus positifs.

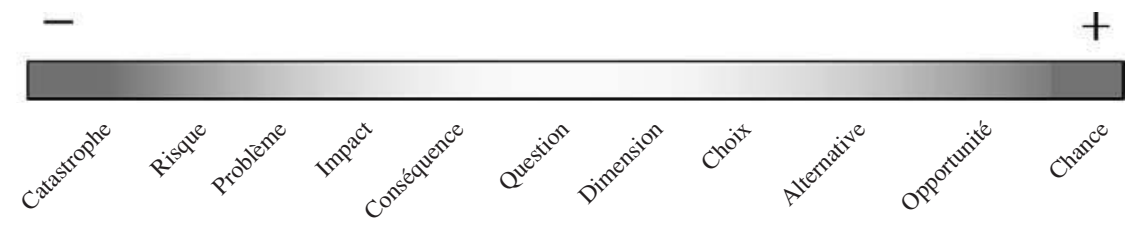


Mais au-delà du jugement de valeur implicite de la notion de risque, une autre propriété tend à renforcer sa dimension stratégique. Il s'agit de sa tendance à impliquer presque mécaniquement une réaction visant l'évitement du risque. Lorsque le risque de dissémination de transgènes est invoqué, une exigence implicite est ce faisant avancée quant à la nécessité de prendre des mesures pour éviter une telle dissémination. Le lien entre risque et mesure d'évitement semble si fort et si organique qu'il ne nécessite aucune justification alors qu'au contraire, lorsque l'on souhaite prendre un risque, un processus de justification est nécessaire qui implique généralement une pesée des risques en fonction des bénéfices.

Certains pourront contester la force de ce lien implicite au motif que, dans l'histoire récente des risques sanitaires et environnementaux, de nombreux risques n'ont pas donné lieu à des réactions d'évitement. Toutefois, il convient de rappeler que l'analyse qui est faite ici se place au niveau de l'argumentation, indépendamment de la véracité des risques invoqués. Le cas de l'encéphalopathie spongiforme bovine (ESB) ou maladie de la vache folle est en ce sens une bonne illustration de ce qui se passe sur le plan de l'argumentation des acteurs ${ }^{2}$. Jusqu'à des stades avancés de développement de cette affaire, les autorités britanniques ont toujours justifié le fait de ne pas prendre de mesures, parfois très simples, en clamant une absence de risques, qu'ils soient hypothétiques ou avérés. Or, elles auraient pu invoquer d'autres motivations, peut-être plus proches de la réalité, telles que des coûts trop élevés pour une agriculture se trouvant déjà en situation difficile. Mais un tel discours n'aurait été recevable ni pour les consommateurs ni pour la population en général. Car admettre l'existence d'un risque, fût-t-il encore hypothétique, pose inévitablement l'exigence de tenter de l'éviter.

Il apparaît ainsi évident que le choix de la notion de risque revêt une dimension stratégique dans un débat: en plus de donner une connotation négative à un argument, le risque y ajoute implicitement l'exigence de mettre en œuvre des mesures d'évitement, ces dernières ne pouvant être contestées qu'en contestant l'existence du risque lui-même.

\subsection{L'identification de risques non reconnus induit la recherche de risques reconnus}

Dans une situation telle que celle relative aux OGM où nombre de questions sont entachées d'incertitudes, le recours à la notion de risque par les divers acteurs impliqués dans le débat public tend à suivre une dynamique particulière.

Dans les cas de risques avérés, ayant trait à des situations peu controversées et à faible degré d'incertitude, le lien temporel entre l'identification d'un risque et la modification du cours des événements est généralement simple, le premier précédant le second. Toutefois, dans des cas plus complexes dans lesquels les enjeux sont multiples, dont les dimensions éthiques et politiques sont importantes, où les responsabilités ne sont pas établies et pour lesquels de nombreux risques demeurent hypothétiques ou non reconnus - comme dans la controverse sur les OGM -

Voir: van Zwanenberg P. et Millstone E., (2001) «»mad cow disease» 1980s-2000: how reassurances undermined precaution», dans Late lessons from early warnings : the precautionary principle 1896-2000, EEA, Copenhagen, pp. 157-167. 
le lien entre l'identification d'un risque et la prise de mesures d'évitement n'est pas forcément linéaire et un effet d'enchaînements et de rétroactions apparaît.

Si l'on considère l'histoire des biotechnologies modernes dans ses grandes lignes, on s'aperçoit que, dès le début, les conséquences possibles de cette technologie ont suscité de nombreuses préoccupations. Les premiers risques invoqués dans l'espace public avaient trait à la santé humaine ainsi qu'aux impacts imprévisibles que des disséminations d'organismes génétiquement modifiés pouvaient avoir sur l'environnement ${ }^{3}$. Le risque de voir l'homme se transformer en apprenti sorcier ou apprenti Créateur ${ }^{4}$, ainsi que le risque de porter atteinte à l'intégrité de la créature en y introduisant des caractères débilitants ont également très vite été évoqués. Enfin, le contexte socio-économique dans lequel les biotechnologies se sont développées a suscité de vives craintes quant à la manière dont elles allaient être utilisées. La domination du marché des plantes transgéniques par quelques grandes entreprises transnationales ainsi que la durée limitée (généralement 20 ans) de la protection par brevets a fait craindre une recherche rapide de profit, au détriment d'une approche de précaution ${ }^{5}$. Pour certains, les OGM sont ainsi rapidement devenus le symbole de la «domination des multinationales», de la «mondialisation néolibérale » et d'une certaine forme de «colonialisme technologique».

Toutefois, étant donné le peu de connaissances disponibles, ces premiers risques étaient par nature vagues et hypothétiques. Ils ne rentraient donc pas dans les catégories de risque pris en compte dans le cadre des évaluations de risques. Ces risques se trouvaient hors du champ de validation ${ }^{6}$ : (1) soit ils n'entraient pas dans le domaine de compétence de disciplines reconnues comme scientifiques et ils n'auraient donc jamais pu être validés, (2) soit ils pouvaient théoriquement l'être puisqu'ils entraient dans le domaine de compétence de ces disciplines, mais ils étaient encore si vagues qu'il aurait fallu des années avant que les recherches scientifiques n'aboutissent à une conclusion permettant de valider ou d'infirmer leur existence.

Ces risques non reconnus correspondent à la définition des menaces ${ }^{7}$ donnée

Ces préoccupations ont notamment été à l'origine de la Conférence d'Asilomar en février 1975.

Voir Howard T. et Rifkin J. (1977) «Who Should Play God? The Artificial Creation of Life and What It Means to the Future of the Human Race?» Dell Publishing, New York.

s Voir dans cet ouvrage l'article de Pascal van Griethuysen: «Pour une approche évolutive de la précaution».

6 L'expression validation d' un risque est utilisée dans cet article au sens du processus par lequel un risque acquiert un statut suffisamment solide pour susciter l'adoption de mesure d'évitement. Elle comprend d'une part la pratique scientifique qui permet de prouver ou de formuler des hypothèses quant à l'existence d'un risque et qui correspond à la pratique officielle et d'autre part les processus participatifs qui prennent place dans le cadre de la controverse et qui permettent de négocier les risques. (Concernant l'expression «négociation des risques», voir Audétat M., Joseph C., Kaufmann A. \& Leresche J.-Ph. (2003) «Usages et limites de l'expertise dans la négociation des risques climatiques et biotechnologiques en Suisse»)

Pour signifier qu'un risque n'est considéré que comme une menace, à savoir qu'il n'est pas reconnu, les acteurs impliqués dans le débat public n'utilisent pas forcément ce terme qui provient de la littérature spécialisée. Comme nous le verrons par la suite, ce qui n'est considéré que comme une menace est généralement qualifié par des termes tels que: «impact», «dimension», «aspect», etc. 
par Ewald ${ }^{8}$. En effet, les menaces ne peuvent être «mises en risques », c'est-à-dire qu'elles ne peuvent être soumises au processus par lequel les experts du risque et en particulier les assurances parviennent à appréhender un risque de manière mathématique ou statistique, en faisant correspondre une valeur monétaire au dommage envisagé, et en en déterminant à la fois les causes et les probabilités d'occurrence.

L'adoption, dans divers textes de loi, de la référence au principe de précaution a toutefois introduit un changement dans la manière d'appréhender certaines menaces. La deuxième catégorie mentionnée ci-dessus regroupant les menaces susceptibles d'être étudiées et en définitive prouvées de manière scientifique ont en effet, grâce au principe de précaution, acquis le statut de risque à part entière. Les risques hypothétiques - leur nouveau nom - peuvent en effet être validés et reconnus; ils sont par ailleurs pris en compte par les instances de régulation et ils peuvent donner lieu à des mesures d'évitement. Nous traiterons plus avant de la question de la précaution mais pour l'instant revenons à la recherche de nouveaux risques.

Parmi les nombreuses menaces évoquées au début de la controverse sur les OGM, le seul risque - un risque hypothétique d'ailleurs - qui ait donné lieu à des mesures d'évitement concerne le risque pour la santé et pour l'environnement résultant de disséminations accidentelles de bactéries recombinantes ${ }^{9}$. Ce risque avait été mis en évidence par les scientifiques et, après un moratoire décrété pendant un an sur toutes les expériences sensibles, un système établissant des normes de confinement physique et biologique a été élaboré lors de la conférence d'Asilomar, en février $1975^{10}$. Les règles établies à Asilomar sont probablement un premier exemple de mesures de précaution par rapport aux OGM, les risques visés par ces mesures n'étant pas clairement identifiés et par nature hypothétiques.

Pour ce qui est des autres menaces, aucune mesure n'a été prise. Alors que l'utilisation en milieu confiné de bactéries recombinantes avait été réglementée, l'arrivée des premières plantes transgéniques a définitivement rendu possible le déconfinement des OGM végétaux ${ }^{11}$. En ce qui concerne la santé, les premières plantes transgéniques ont été mises sur le marché dès 1989, en dépit du peu de recherches effectuées concernant les risques éventuels ${ }^{12}$. De plus, en ce qui concerne le risque de voir les scientifiques se transformer en apprentis sorciers, un

8 Cette expression de François Ewald dans «L'Etat providence» est citée par Claude Gilbert dans «La fin des risques?», in Quaderni, La revue de la communication, n 48, 2002, pp. 111-120.

$9 \quad$ Il s'agit bien entendu de la deuxième catégorie de menace, celles qui sont devenues des risques hypothétiques suite à l'introduction du principe de précaution.

10 Pour plus de détails à ce sujet, voir: Krimsky S. (1992), «Recombinant DNA research», in Controversy, Politics of technical decisions, Third Edition, Dorothy Nelkin, editor, Sage Publications, USA.

${ }^{11}$ Les premiers essais d'OGMs en plein champ ont eu lieu dès 1986 (Source: James C. (1997) «Global status of transgenic crops in 1997 », ISAAA Briefs No.5, ISAAA: Ithaca, NY).

12 Les recherches concernant les risques pour la santé, notamment à travers l'alimentation, sont restées jusqu'à aujourd'hui très limitées. Voir à ce sujet: Butler D. and Reichhardt T. (1999), «Long-term effect of GM crops serves up food for thought», Nature, Vol. 398, pp.651-653. Voir également: Pusztai A. (2002), «GM food safety: scientific and institutional issues », Science as Culture, Vol. 11, pp. 69-92. 
arrêt complet et peut-être définitif de toute recherche en génie génétique aurait été nécessaire, ce qui n'était bien entendu pas envisageable compte tenu des promesses de cette technologie à la fois sur les plans médical, agronomique et surtout économique. Quant au risque de voir la recherche de rentabilité économique prévaloir sur une approche de précaution, la large diffusion des OGM et leur consommation, notamment aux Etats-Unis, dès le milieu des années 90 montre que les mesures nécessaires à prévenir ce risque n'ont pas été prises.

Afin de donner plus de substance et de crédibilité à ces menaces, un travail d'investigation a donc été entrepris, par des scientifiques d'une part, mais également par des non spécialistes faisant partie des acteurs de la controverse. Des risques plus précis ont été identifiés et des hypothèses plus détaillées ont été formulées. Le travail de recherche des scientifiques a été complémentaire au travail de recherche des non spécialistes, ces derniers ayant eu essentiellement trois rôles: celui de lanceur d'alerte ${ }^{13}$, celui de relais des résultats de la recherche scientifique et celui de constructeurs de nouvelles hypothèses explicatives.

Le lien temporel entre l'identification des risques et les mesures d'évitement n'a donc pas été linéaire. L'identification des premières menaces n'ayant pas abouti à une prise de mesures d'évitement, la nécessité de trouver des risques plus plausibles et plus fondés est apparue. Par effet de rétroaction, de nouvelles recherches concernant les risques possibles ont donc été entreprises. Une fois identifiés, ces nouveaux risques - qui se trouvent cette fois à l'intérieur du champ de validation du fait leur degré de précision mais également du fait du domaine auquel ils se réfèrent - ont non seulement permis de mieux justifier la prise de mesures demandée dès le commencement, mais également de renforcer la conviction de ces acteurs quant à la nécessité de modifier le cours des évènements, induisant de nouvelles investigations et ainsi de suite. C'est cet effet d'enchaînement et de rétroaction positive qui constitue la dynamique particulière qui accompagne la controverse et le recours stratégique à la notion de risque. Face à des menaces qui ne peuvent être validées et qui ne sont donc pas reconnues par les instances de régulation, c'est un véritable «filet à risques » qui est déployé par investigations successives, jusqu'à l'identification de risques suffisamment précis et étayés pour être validés et permettre une modification du cours des évènements par la prise de mesures adéquates. Cet effort de recherche porte naturellement sur la santé et l'environnement qui sont les seuls objets valorisés ${ }^{14}$ qui peuvent être étudiés par des disciplines scientifiques.

La recherche de nouveaux risques répond donc à deux nécessités distinctes: (1) la première est d'éviter que les risques reconnus (risques avérés et hypothétiques) ne se réalisent, et (2) la deuxième est celle d'éviter la réalisation des risques non reconnus (menaces) qui ne peuvent être validés et qui doivent ainsi être prévenus de manière indirecte. Les menaces ont donc une fonction de motivation pour identifier des nouveaux risques qui pourront être validés. Elles jouent ainsi un rôle dans l'ouverture de nouvelles pistes de recherche. L'alerte concer-

13 Chateauraynaud F., Torny D. (1999), «Les sombres précurseurs: une sociologie pragmatique de l'alerte et du risque », Paris, éd. de l'Ecole de hautes études en sciences sociales, p. 80.

14 La notion d'objet valorisé est développée dans la deuxième partie de cet article. De plus, le champ des risques reconnus y sera défini en fonction des deux paramètres mentionnés ici, à savoir les objets valorisés par la société et les disciplines considérées comme scientifiques. 
nant le papillon Monarque ${ }^{15}$ par exemple, a remis en question le caractère inoffensif présumé du pollen de maïs Bt. De récentes études ont finalement permis de démontrer que, bien que la majorité des maïs cultivés aux Etats-Unis n'ait pas d'effet négatif sur le papillon Monarque, la variété de maïs Bt-176, qui contient 40 fois plus de toxine $\mathrm{Bt}$ que les autres, influe négativement sur les larves de ce lépidoptère ${ }^{16}$.

Toutefois, le double propos de cette recherche de nouveau risques reconnus est également susceptible de générer des situations paradoxales, tel que le montre l'exemple des gènes marqueurs de résistance aux antibiotiques ${ }^{17}$. Le risque invoqué concernant ces gènes marqueurs a trait à la possibilité qu'un tel gène soit transmis à des bactéries du sol ou de l'intestin ce qui, dans le cas où ces bactéries seraient pathogènes pour l'homme, viendrait augmenter les problèmes de résistances aux antibiotiques rencontrés en médecine humaine. En examinant cette question de manière étroite, c'est-à-dire en focalisant uniquement sur les plantes transgéniques, il est tout à fait admissible de considérer que la transmission du gène marqueur de résistance aux antibiotiques, bien que n'ayant jamais été démontrée in vivo, constitue tout de même un risque hypothétique qui nécessite l'adoption de mesures de précaution. Par contre, du moment que l'on considère le problème des résistances aux antibiotiques dans son ensemble, une telle argumentation ne résiste pas à l'épreuve de la cohérence ${ }^{18}$. Le problème des résis-

15 L'alerte concernant les effets du pollen Bt sur le papillon Monarque a débuté par la publication d'un article dans Nature (Losey J. E., Rayor L. S., Carter M. E. (1999) « Transgenic pollen harms monarch larvae», Nature, Vol. 399, p. 214.) faisant état d'une mortalité accrue des larves de ce papillon lorsqu'elles s'étaient nourries avec des feuilles recouvertes de pollen Bt. Cette nouvelle fut reprise et diffusée par les réseaux opposés aux OGM, engendrant un véritable battage médiatique. Dans ce cas, les acteurs sociaux ont joué le rôle de relais des résultats de la recherche scientifique. Il est raisonnable en outre de considérer que la diffusion de cette information auprès des médias a contribué a susciter d'ultérieures recherches visant à prouver ou infirmer ce risque qui, au moment de l'alerte, n'était encore qu'hypothétique.

16 Zangerl A. R., McKenna D., Wraight C. L., Carroll M., Ficarello P., Warner R. and Berenbaum M. R. (2001), «Effects of exposure to event 176 Bacillus thuringiensis corn pollen on monarch and black swallowtail caterpillars under field conditions », Proc. Natl. Acad. Sci., Vol. 98, pp.1190811912.

Stanley-Horn D. E., Dively G. P., Hellmich R. L., Heather R. M., Sears M. K., Rose R., Jesse L.C., Losey J. E., Obrycki J. J. and Lewis L. (2001), «Assessing the impact of Cry1Ab-expressing corn pollen on monarch butterfly larvae in field studies » Proc. Natl. Acad. Sci., Vol. 98, pp.11931-11936.

Hellmich R. L. and Siegfried B. D. (2001), in Genetically Modified Organisms in AgricultureEconomics and Politics, ed. Nelson, G. C. (Academic, London), pp. 283-289.

17 L'utilisation de ces gènes marqueurs est rendue nécessaire du fait de la faible efficacité du processus de transformation des plantes. Un moyen de reconnaître les plantes effectivement transformées doit donc être utilisé. Le gène marqueur permet précisément de procéder à cette sélection car seules les plantes transformées sont capables de survivre à l'application de l'antibiotique.

18 L'exigence de cohérence dans les mesures prises face à des risques similaires est considérée comme faisant partie intégrante de la mise en œuvre du Principe de Précaution (PP). Le PP ne peut en effet venir justifier des mesures discriminatoires. Voir: Godard O., «De nouvelles donnes, une nécessaire nouvelle approche », Maîtrise des risques - Prévention et principe de précaution, Actes du Colloque du 6 novembre 2001, Institut Pasteur, pp. 62-63. Voir également: Commission des Communautés Européennes (2000), «Communication de la Commission sur le recours au principe de précaution», Bruxelles, Url: http://europa.eu.int/eur-lex/fr/com/cnc/2000/com2000_0001 fr01.pdf, pp. 18-19. 
tances aux antibiotiques est apparu suite à l'utilisation massive d'antibiotiques, depuis une cinquantaine d'années, à des fins non seulement thérapeutiques en médecine humaine et vétérinaire, mais également comme additifs alimentaires dans l'élevage de bétail et de poissons ainsi que sur certaines cultures. Ce problème ainsi que ses causes - la pression de sélection due à la présence de l'antibiotique - étaient donc connus bien avant l'ère des plantes transgéniques. Pourtant, des mesures efficaces visant une réduction drastique de l'emploi d'antibiotiques ne se mettent en place que très lentement: que ce soit en agriculture ou en médecine, les antibiotiques continuent à être utilisés de manière excessive. Que dire dans un tel contexte de la cohérence d'un discours qui s'attaque aux plantes transgéniques au motif qu'elles portent un gène de résistance aux antibiotiques, mais qui ne mentionne nulle part le risque incommensurablement plus grand et mieux étayé lié à l'emploi abusif d'antibiotiques?

Cette incohérence dans la revendication de mesures d'évitement met en lumière le fait que le risque de développement de résistances aux antibiotiques ne constitue pas dans ce cas la préoccupation principale, mais que la mise en évidence de ce risque répond bien plus à la nécessité d'éviter la réalisation des menaces qui ne sont pas prises en compte dans le processus d'évaluation des risques. La recherche de nouveaux risques répond donc parfois plus à la nécessité de motiver la prise de mesures pour empêcher la réalisation de certains risques, généralement des menaces - et donc non pris en compte dans le cadre d'une évaluation des risques - que de la préoccupation pour chacun des risques identifiés.

Mais un autre point doit être mentionné concernant la distinction risque menace. En effet, si cette classification permet de distinguer utilement entre des risques qui peuvent être validés et reconnus et d'autres risques qui ne peuvent pas l'être, elle avalise cependant les raisons sur lesquelles se fonde cette distinction. Or, le propos des acteurs de la société civile impliqués dans cette recherche de nouveaux risques semble précisément être de battre en brèche cette distinction et de faire valider et reconnaître ces risques qui ne sont encore considérés que comme des menaces. En ce sens, le fait d'étendre l'emploi de la notion de risque à toutes les menaces revient à en faire un emploi qui peut être qualifié de stratégique puisqu'il vise précisément la remise en cause de cette distinction et l'accession de toutes les menaces au statut de risques pouvant être validés et reconnus. La distinction risque - menace ne saurait donc être considérée comme objective, mais plutôt comme le reflet de présupposés normatifs (les méthodes scientifiques comme seules méthodes permettant de déterminer les risques à prendre en compte) qui sont aujourd'hui remis en question.

\subsection{Objection concernant l'extension de la notion de risque}

Ces éléments nous amènent à présent à considérer l'objection qui est faite par certains auteurs ${ }^{19}$ quant à l'extension de la notion de risque. Cette objection ne

Voir dans cet ouvrage: van Griethuysen P., «Pour une approche évolutive de la précaution».Voir également Callon M., Lascoumes P., Barthes Y. (2001) «Agir dans un monde incertain, essai sur la démocratie technique», Seuil, pp. 37-40. Ces derniers proposent d'utiliser la notion d'incertitude plutôt que de risques. Or, si cette notion permet précisément de mettre en évidence le caractère incertain (sic!) de certaines situation, sa valence de mobilisation est en revanche faible: elle 
porte pas sur l'élargissement du champ des risques reconnus par les instances de régulation - point de vue que les auteurs tendent à soutenir - mais sur le fait d'utiliser le terme «risque» pour désigner ces possibles impacts négatifs. Le principal argument qui est avancé concerne le fait que la notion de risque est étroitement associée à celle de choix rationnel. Elle impliquerait une connaissance parfaite des scénarios possibles, des probabilités d'occurrence du dommage ainsi que de la gravité de ce dommage. Cette connaissance complète devrait ensuite servir de base à la décision.

Les incertitudes multiples qui entourent le développement d'une technologie telle que les OGM imposent naturellement de prendre ses distances par rapport à une telle approche. Toutefois, on peut se demander s'il est vraiment indispensable que la notion de risque soit abandonnée ou plutôt réservée aux uniques risques avérés ${ }^{20}$ au motif qu'elle ne saurait s'extraire du modèle d'évaluation et de gestion des risques inspiré par la théorie dite du choix rationnel. Bien que l'on puisse partager dans une certaine mesure les craintes exprimées par ces auteurs, nombre d'éléments semblent montrer que, grâce à sa grande flexibilité, la notion de risque s'est déjà en partie affranchie de cet attachement à une rationalité jugée trop limitante. Le simple recours de plus en plus fréquent à la notion de risque hypothétique semble en être une bonne démonstration.

En conclusion, s'il convient de ne pas minimiser les désavantages de la notion de risque - que ce soit son association à la notion de choix rationnel ou encore le fait de générer une argumentation focalisée sur les éventualités négatives plutôt que positives - il semble toutefois judicieux de ne pas limiter son utilisation aux seuls risques avérés avant d'avoir pu examiner dans quelle mesure l'usage stratégique de cette notion par les acteurs sociaux peut se justifier ou non.

\section{LA NOTION DE RISQUE POUR LES INSTANCES DE DÉCISION (OU LE SYSTÈME CLASSIQUE D'ÉVALUATION DES RISQUES)}

Au-delà de sa valeur stratégique pour les acteurs sociaux, le risque a également un statut particulier pour les instances chargées d'encadrer le développement des biotechnologies. Qu'elles soient juridiques, politiques ou administratives, les instances de décision et de régulation accordent une reconnaissance presque exclusive au paramètre risque en tant que facteur pouvant justifier la modification, l'arrêt ou le ralentissement du développement des biotechnologiques. S'il n'y a pas de risque, l'interdiction de commercialiser un produit, l'arrêt des recherches dans un domaine prometteur ou encore le refus

ne fait référence à aucun danger et à fortiori à aucun dommage, tout comme elle ne laisse entrevoir aucune opportunité ou amélioration. Son caractère plutôt neutre la réserve à un emploi détaché qui est le fait des chercheurs en sociologie des sciences, mais non pas à un emploi engagé dans le feu de la controverse. Définir quelque chose comme une incertitude est rarement un enjeu dans le débat public alors que le définir comme un risque en constitue souvent tout l'enjeu...

${ }^{20}$ En suivant le raisonnement des auteurs défavorables à l'extension de la notion de risque, les risques hypothétiques, bien que faisant à présent partie des risques reconnus, ne devraient logiquement pas être considérés comme des risques à part entière, mais plutôt comme des incertitudes, des menaces, etc. 
d'autoriser un essai de plante transgénique en pleins champ ont de fortes chances d'apparaître comme des décisions arbitraires et injustifiées.

Les instruments de régulation (traités internationaux, droit communautaire, législations nationales, procédures d'autorisation, droit de recours, etc.) tendent ainsi à subordonner la limitation de la diffusion des OGM (recherche, dissémination, commercialisation, consommation) à la présence de risques. La présence d'un risque - avéré ou pour le moins hypothétique - constitue le plus souvent la seule raison valable pour exercer une influence sur le développement d'une technologie. En effet, la diffusion d'une technologie et les gains en termes économiques qui en découlent restent à l'heure actuelle la règle dominante et implicite; l'exception à cette règle - la limitation ou la modification de la trajectoire de la technologie - n'étant admise que pour des bonnes raisons, c'est-à-dire la présence de risques. Cet état de fait est enraciné à la fois dans la façon dont l'indépendance de la science et de la technologie est implicitement reconnue et dans l'adhésion de plus en plus étroite des systèmes juridiques nationaux et internationaux aux règles du libre-échange économique ${ }^{21}$.

A noter toutefois que des principes éthiques tels que la liberté de choix des consommateurs $^{22}$ ou encore le respect de l'intégrité des organismes vivants ${ }^{23}$ ont récemment été inscrits dans le cadre légal de divers pays, notamment européens. Ces principes d'ordre général, auxquels le développement des biotechnologies doit se soumettre introduisent donc une rupture dans l'approche scientiste qui a prévalu jusqu'alors dans la gestion des biotechnologies vertes ${ }^{24}$ et ils peuvent à ce titre être considérés comme un premier pas vers une définition élargie des critères d'évaluation de cette technologie.

Mais il apparaît rapidement que tous les risques n'ont pas la même valeur et le même poids pour justifier des mesures de contrôle de la technologie. Qu'est-ce qui différencie les risques très divers apparaissant dans le débat public? Quels sont ceux qui sont reconnus comme pertinents pour limiter le développement des biotechnologies? Pourquoi dans l'optique de l'évaluation des risques classique certains sont-ils effectivement considérés comme des risques et requirent l'adoption de mesures de précaution ou de prévention et pourquoi d'autres ne le sont pas? Pourquoi le risque de franchir un échelon supplémentaire dans l'instrumentalisation de la nature par exemple n'est-il pas considéré comme un risque au même titre que celui de voir se développer des résistances chez les insectes suite à la culture de plantes transgéniques Bt?

${ }^{21} \quad$ Il est donc fort logique que ces règles du jeu aient été prises en compte par les acteurs impliqués dans le débat public - de manière consciente ou non - et que de ce fait toute tentative d'influer sur le cours d'un développement technologique tende à se traduire par une recherche des risques, qui seuls en constituent une justification.

22 Voir: directive 2001/18/CE, règlement 258/97, règlement (CE) 1139/98, règlement (CE) 49/2000, règlement (CE) 50/2000 du droit communautaire. Voir également: droit suisse, Loi fédérale sur l'application du génie génétique dans le domaine non humain, (art. 1, al. 2, lett. d; art. 7)

23 Voir: droit suisse, Constitution fédérale, art. 120, al.2. et Loi fédérale sur l'application du génie génétique dans le domaine non humain, art. 8.

${ }^{24}$ Il s'agit des biotechnologies appliquées aux domaines de l'agriculture et de l'alimentation. Elles sont qualifiées de «vertes» car, pour l'instant, les plantes transgéniques figurent au premier chef des applications commercialisées dans ces domaines, exception faite des micro-organismes utilisés dans l'industrie agro-alimentaire. 
Afin de mieux comprendre la différence entre les risques reconnus et ceux qui ne le sont pas, nous allons reprendre la distinction sur deux plans introduite dans le tableau en annexe 1. Le premier plan a trait au type d'objet susceptible de subir le dommage. Il faut en effet que la société accorde suffisamment de valeur à cet objet pour que sa destruction soit véritablement considérée comme une éventualité à éviter. Il est ainsi évident que le risque de porter atteinte à la population de pyrale (un insecte ravageur du maïs) ne sera pas un risque reconnu étant donné que l'objet susceptible de subir le dommage - la pyrale - n'est nullement valorisé, bien au contraire. Le deuxième plan sur lequel il est possible de discriminer les risques reconnus de ceux qui ne le sont pas est celui de la méthode de validation qui permet de fournir soit la preuve soit une hypothèse fondée de l'existence d'un risque. La seule méthode d'investigation reconnue comme valable est la méthode scientifique pratiquée dans le cadre des disciplines considérées comme faisant partie de la science ${ }^{25}$. Chacun de ces deux critères étant une condition sine qua non, cela revient à dire que seuls sont reconnus les risques - hypothétiques ou avérés - pouvant induire un impact négatif sur un objet valorisé par la société et dont l'existence - ou la vraisemblance de l'hypothèse - peut être établie selon une méthode de validation reconnue, la méthode scientifique. C'est l'intersection entre l'objet valorisé et la méthodologie reconnue qui définit le champ des risques pris en compte dans le cadre de l'évaluation des risques classique.

\subsection{Objets valorisés}

Les risques mentionnés par les acteurs sociaux montrent que des objets de nature très différente peuvent être valorisés. Le terme «objet» ne recouvre donc pas ici que des entités matérielles, mais également des entités immatérielles ou plutôt hybrides. La liberté de choix des consommateurs par exemple est un objet valorisé, de nature immatérielle, qui dans l'éventualité où un étiquetage des produits OGM ne serait pas garanti, pourrait subir un dommage, à savoir une diminution voire une élimination de cette faculté de choisir librement. L'intégrité des organismes vivants ${ }^{26}$ est un autre objet valorisé, de nature plutôt hybride, qui pourrait être affecté de manière négative dans le cas où des traits débilitants et

25 L'acception donnée ici, de même que dans la suite de cet article, aux termes «science» ou «scientifique » est celle, très limitée, qui a été généralement adoptée par les instances de régulation et qui correspond aux sciences *naturelles. En effet, bien que les législations, nationales ou internationales, fassent référence à la «science», à la «méthode scientifique», ou encore à la «sound science» (en anglais), en omettant généralement de préciser de quel type de science il s'agit, le contexte et les raisons pour lesquelles est invoqué le recours à la «science» montre clairement qu'il s'agit des sciences naturelles et non pas des sciences sociales, économiques ou humaines. Comme le fait remarquer Doaa Abdel Motaal ( Science, Risk Assessment and WTO Rules; The Role of Scientific Expert Consultation», Thèse de doctorat, Institut Universitaire du Développement, Décembre 2003) à propos du sens du terme «science» dans l'accord sur l'application de mesures sanitaires et phytosanitaires (SPS) de l'Organisation Mondiale du Commerce: «[...] in reading the negociating history of both agreements [SPS and TBT] one emerges with a clear sense that negociators had the natural, rather than the social sciences in mind. This is made particularly evident by the fact that lengthy discussions were held on the role that other international institutions, working in the natural science domain, such as the Codex Alimentarius, would play.».

26 L'intégrité des organismes vivants est une notion qui a été récemment introduite dans la constitution suisse (art. 120.2). 
engendrant des souffrances inutiles seraient introduits par génie génétique dans un animal. La nature hybride de cet objet est due au caractère proprement physique de la modification génétique d'une part et à l'abstraction qui confère une certaine moralité à un état d'être particulier considéré comme intègre pour un organisme vivant. Enfin, l'indépendance des paysans vis-à-vis de l'agro-industrie, le libre accès aux semences, la possibilité de ressemer une partie de sa récolte, le maintient des ressources génétiques dans le domaine public sont tous des exemples d'objets valorisés de nature hybride qui ont trait plus ou moins directement à la sphère des droits socio-économiques.

En outre, une partie des objets mentionnés dans le débat public fait appel à des projections particulières dans le temps: ce qui est menacé n'est pas un objet existant dans le présent tel que la biodiversité par exemple, ou la possibilité de choisir une alimentation sans OGM; ce qui est menacé est la réalisation d'une situation future recherchée et considérée comme bonne ou meilleure que la situation présente. Une partie importante de la mobilisation contre les OGM a ainsi pour toile de fond un combat pour la réalisation d'une société dont les caractéristiques saillantes seraient: le respect de l'environnement, le respect des droits de l'homme, l'élimination de la faim, de la pauvreté, des inégalités, la promotion d'un autre type de développement (développement durable par exemple) ou encore le respect de la diversité des cultures ${ }^{27}$.

Ces représentations futures sont donc bien des projets de société que certains acteurs souhaiteraient voir se réaliser, une société idéale vers laquelle ils veulent tendre. Les développements technologiques qui, de par leurs caractéristiques, semblent incompatibles avec le projet de société d'un acteur seront interprétés par ce dernier comme un risque pouvant compromettre la réalisation de cette situation future. Le projet de société correspond ainsi à l'objet valorisé susceptible de subir un dommage. Concrètement, parmi les risques pouvant affecter la réalisation des projets de société auxquels adhèrent nombre d'opposants aux OGM, on compte notamment le risque de pillage des ressources génétiques de pays du Sud grâce au système des brevets, le risque de domination accrue des multinationales ainsi que le risque de «marchandisation» (de stricte soumission aux impératifs économiques) de domaines complexes et surtout vitaux tels que l'agriculture et l'alimentation.

Ce type de risques naît de la confrontation entre un projet de société dominant et implicite qui est incarné par la technologie mise en cause et des projets de société alternatifs. Dans le cas des OGM, ces menaces ont été pour une bonne part dans la motivation qui a conduit à la recherche de nouveaux risques, susceptibles d'être validés. Toutefois, ces menaces ne pouvant être éliminées sans une remise en cause et une modification du projet de société dominant, on comprend dès lors mieux la virulence de l'opposition aux biotechnologies et son insensibilité à

Tous les acteurs opposés aux OGM n'ont pas une vue strictement identique de la société qu'ils voudraient voir se réaliser, et les caractéristiques qui sont mises en évidence ici n'ont qu'une valeur d'exemple. De plus, certaines personnes, notamment des scientifiques, partagent - en tout cas partiellement - les idéaux des opposants aux OGM, tout en étant favorables à cette technologie. Cette variation radicale dans l'interprétation des conséquences des biotechnologies met en évidence une fois de plus la nécessité d'une expertise indépendante concernant les risques socioéconomiques des OGM. 
d'éventuelles réductions des risques reconnus - à savoir les risques pour la santé et l'environnement.

Tel fut le cas en Suisse lors des trois demandes de disséminations expérimentales déposées et en particulier dans le cas de la dernière d'entre elles qui concerne le blé transgénique KP4 résistant à la carie, une maladie fongique ${ }^{28}$. En effet, de nombreuses mesures ont été prises pour réduire considérablement les risques pour l'environnement: filet imperméable au pollen, semis de bordure et distances de sécurité pour éviter les disséminations de pollen, destruction de tout le matériel une fois l'essai terminé afin qu'aucune de plantes de blé transgénique ne puisse persister dans le champ, surface de l'essai extrêmement réduite $\left(8 \mathrm{~m}^{2}\right)$, contrôle quotidien du champ par un scientifique, herbicide total disponible à proximité en cas d'incident ${ }^{29}$. Or, ces dispositions n'ont nullement permis de rendre moins virulente l'opposition à cet essai, bien au contraire. La raison en est que les risques visés par ces mesures étaient tous des risques reconnus ne prenant en compte que les éventuels dommages à l'environnement. Les menaces, à savoir tous les risques non reconnus ${ }^{30}$ n'ayant nullement été traitées, l'opposition à cet essai est restée intacte.

Enfin, la santé et l'environnement sont les deux objets, de nature plutôt matérielle qui, contrairement aux autres, sont non seulement valorisés par nombre d'acteurs, mais sont également reconnus par les instances de régulation. Ainsi, comme mentionné précédemment, les éventuels dommages à ces deux objets sont les seuls à justifier, aux yeux des instances de régulation, une limitation du développement des biotechnologies. La liberté de choix des consommateurs ainsi que l'intégrité des organismes vivants sont certes des objets valorisés permettant de limiter le développement des biotechnologies, mais d'une manière qui reste très limitée. En effet, la nécessité de préserver la liberté de choix des consommateurs ne peut justifier tout au plus qu'un étiquetage des produits contenant des OGM ainsi que la séparation des filières OGM - non OGM. Quant au respect de l'intégrité de la créature, cette exigence fait essentiellement référence aux souffrances inutiles infligées aux animaux et ne s'applique de ce fait que difficilement aux plantes transgéniques. L'effort de recherche qui, par enchaînement et rétroaction, abouti à identifier de nouveaux risques tend ainsi à se focaliser sur les deux seuls objets - la santé et l'environnement - capables de justifier une modification en profondeur de la trajectoire de la technologie et de répondre finalement aux préoccupations les plus profondes des acteurs sociaux - l'évitement des menaces ou risques non reconnus par les instances de régulation.

28 Voir dans cet ouvrage: Perret H., Bordogna Petriccione B., Kaufmann A., Audétat M., Joseph C. «Disséminations exérimentales d'OGM en France et en Suisse: évolution du débat public».

${ }_{29}$ CFSB (2001), «Prise de position de la CFSB concernant la demande B00003 de l'Institut de biologie végétale de l'EPFZ. Comportement des variétés de blé transgénique KP4 en plein champ.», url: http://www.buwal.ch/stobobio/cfsb/pdf/Prise_de_pos.pdf

3o Par exemple: danger pour l'agriculture suisse et inconsistance avec la politique agricole fédérale, risque que cet essai n'ouvre la porte aux plantes transgéniques sans possibilité de retour en arrière, risque de s'engager dans une voie contraire et incompatible avec le développement durable ou encore risque découlant d'un lien soupçonné entre entreprises privées et recherche publique. 


\subsection{La science, seule méthode d'investigation / validation reconnue}

La définition d'une pratique ou d'une méthodologie reconnue comme pouvant prouver l'existence d'un risque est une réponse à la question de savoir comment procéder pour identifier, parmi les risques nombreux et diversifiés invoqués par les acteurs sociaux, ceux qui sont pertinents et objectifs. La science, de par l'aura d'autorité dont elle bénéficie en Occident, reste le moyen de connaissance du monde considéré comme le plus objectif et le plus fiable. Il en découle naturellement que toute recherche visant à distinguer un risque sans fondement réel d'un risque effectif s'appuie sur la science et sur la méthode scientifique pour arriver à ses fins. Distinguer entre l'arbitraire et le justifié, telle est donc la tâche de la science en regard des restrictions qui pourraient affecter les paradigmes du progrès technologique et du libre-échange économique. De ce fait, tous les risques qui ne peuvent être identifiés et validés scientifiquement sont de facto exclus et ils ne sont pas pris en compte par les instances de régulation.

Dans le tableau en annexe 1, nombre de risques apparaissent avec la mention «autres» dans la colonne relative à la méthode d'identification/validation. Ces «autres» ne sont certes pas homogènes mais ce qui les caractérisent en premier lieu dans ce contexte, c'est leur non appartenance au domaine scientifique et donc le fait que les risques qui devraient être identifiés et validés selon ces méthodes sont des risques non reconnus.

Il convient à présent de noter que, au-delà de la distinction scientifique - non scientifique, le choix des disciplines scientifiques qui seront amenées à contribuer à l'analyse des risques est également susceptible d'influencer le type de risques identifiés. En effet, les disciplines pouvant contribuer à mettre en évidence les risques sanitaires et environnementaux liés aux biotechnologies sont nombreuses: génétique, biologie moléculaire, protéomique, biologie des populations, toxicologie, écotoxicologue ou encore épidémiologie. Chacune de ces disciplines a son propre objet d'étude, son échelle d'observation à la fois spatiale (ADN, cellule, organisme, écosystème) et temporelle (seconde, minute, année, vie d'un individu, vie d'une espèce, évolution toute entière). Elles mobilisent des théories et des hypothèses particulières, et leur instrumentation, leurs modèles et leurs dispositifs expérimentaux varient ${ }^{31}$. Ce cadre à la fois théorique et pratique fixe à chaque discipline ses propres limites au-delà desquelles elle perd toute pertinence. Ainsi, la biologie moléculaire serait de peu d'utilité pour déterminer l'impact à long terme sur la santé résultant de la consommation de plantes transgéniques, mais elle serait en revanche indispensable pour s'assurer que seule une copie du transgène a été introduite dans la plante transformée. Chaque type de risques est pour ainsi dire «adapté » à une discipline particulière au moyen de laquelle il peut être identifié et démontré de manière optimale.

Concrètement, ce point tout à fait crucial pour la gestion des biotechnologies - le choix des disciplines scientifiques contribuant à l'analyse des risques - se joue en premier lieu sur le plan de l'expertise et en particulier de la composition des comités d'experts. Aux experts sélectionnés correspondront des risques iden-

Voir: Bonneuil C. (2002), «La question des cadrages de l'expertise en situation d'incertitude», Maîtrise des risques - Prévention et principe de précaution, Actes du Colloque du 6 novembre 2001, Institut Pasteur, pp. 81-94. 
tifiés qui seront essentiellement fonction de la diversité des disciplines représentées dans le comité ${ }^{32}$. A travers un phénomène qui est appelé par les sociologues cadrage de l'expertise "sont définis - souvent implicitement - les impacts, les problèmes, les scénarios qui sont à prendre en compte par rapport à ceux qui ne sont pas à prendre en compte [...] [Le cadrage de l'expertise] est ce qui définit ce que le collectif expert va considérer comme une zone d'ombre ou d'ignorance et le reconnaître comme tel. [...] Le cadrage porte aussi sur le type de recherches que le collectif va considérer comme pertinentes pour répondre à ces zones d'ombre par rapport aux types de recherches qu'on va considérer (ou non) pour répondre à ces questions $\gg^{33}$. Un exemple de réajustement du cadrage de l'expertise a eu lieu notamment au sein de la Commission du génie biomoléculaire (CGB) en France, qui est la commission d'experts chargée d'évaluer les risques pour l'environnement et la santé publique liés à la dissémination volontaire d'OGM soit à des fins de recherche et développement ou de mise sur le marché. Christophe Bonneuil montre que la CGB, qui a été créée en 1993, a été dominée par des experts issus de la biologie moléculaire jusqu'en 1998, date à laquelle sa composition a été revue afin de mieux représenter des disciplines tels que la biologie des populations, l'entomologie ou l'agronomie. Cette redéfinition du cadrage de l'expertise est intervenue suite aux critiques adressées à l'approche trop étroite d'une évaluation des risques dominée par le paradigme moléculariste: «Schématiquement, pour des chercheurs issus de la biologie moléculaire [...], la maîtrise des risques liés aux OGM passe d'abord par la propreté et l'élégance de la construction génétique, par la réalisation d'une séquence linéaire d'ADN qui soit stable, bien connue, sans fragments superflus $»^{34}$. Ainsi, contrairement aux certitudes affichées au début des années 90 concernant l'absence de risques liés à la dissémination de transgènes via le pollen, des études, en particulier dans les domaines de la génétique et de l'écologie des populations, ont montré qu'il y a bien un risque d'hybridation avec des plantes sauvages apparentées dans le cas du colza transgénique ${ }^{35}$, aboutissant au décret d'un moratoire sur toutes cultures commerciales de colza génétiquement modifiés en France dès $1998^{36}$.

Ainsi, s'il est convenable d'admettre qu'à chaque discipline correspond un spectre de risques qui pourront être identifiés et évalués, il est alors nécessaire de reconnaître le lien existant entre les disciplines prises en compte dans le cadre de l'évaluation des risques et les risques mis en évidence à travers ce processus. L'évaluation des risques devrait par conséquent impliquer un éventail aussi large

32 D'autres facteurs peuvent également entrer en ligne de compte telle que la prédisposition des experts à considérer les limites de leur propre discipline, les potentialités d'autres méthodes d'investigation ainsi que la part d'incertitude et d'ignorance relative à la situation.

33 Bonneuil C. (2002), «La question des cadrages de l'expertise en situation d'incertitude», Maîtrise des risques - Prévention et principe de précaution, Actes du Colloque du 6 novembre 2001, Institut Pasteur, p. 90.

34 Bonneuil C. (2002), «La question des cadrages de l'expertise en situation d'incertitude», Maîtrise des risques - Prévention et principe de précaution, Actes du Colloque du 6 novembre 2001, Institut Pasteur, p. 88.

35 Chèvre A-M., Eber F. and Renard M. (1998), «Plantes génétiquement transformées: quels risques de dissémination de gènes dans l'environnement?», in Organisme Génétiquement Modifié à l'INRA, Environnement, Agriculture et Alimentation, INRA. Url: http://www.inra.fr/Internet/ Directions/DIC/ACTUALITES/DOSSIERS/OGM/OGM.htm

36 Chèvre A-M., Eber F. and Renard M. (1998), op. cit., p. 85. 
que possible de disciplines scientifiques. A noter que, dans le cas où des risques hypothétiques ont été envisagés mais que, en raison de leur nouveauté, aucune discipline ne se trouve adaptée à leur spécificité, il est raisonnable de s'attendre à ce que de nouveau dispositifs expérimentaux, de nouveaux instruments ainsi qu'une échelle d'observation différente soient développés. Mais au-delà des risques hypothétiques, cette créativité dans la recherche de nouvelles méthodes d'investigation s'avère nécessaire en particulier dans les cas d'ignorance rationnellement orienté ${ }^{37}$ tel que celui de l' «ADN parasite» (junk DNA en anglais) où les risques n'ont même pas encore été envisagés, mais où le manque de connaissance dans ce domaine directement affecté par la transgénèse impose une recherche active des conséquences possibles.

En conclusion de cette partie concernant la notion de risque pour les instances de décision, on peut donc dire que le champ des risques reconnus résulte de l'intersection entre l'objet valorisé et la méthode d'identification/validation reconnue et que par conséquent, en ce qui concerne les OGM, cette intersection correspond aux risques environnementaux et sanitaires qui peuvent être prouvés ou conjecturés scientifiquement.

\section{FONDEMENTS DE DEUX ACCEPTIONS DIFFÉRENTES DE LA NOTION DE RISQUE}

\subsection{Quelles justifications pour une acception étroite des risques reconnu?}

Après avoir considéré l'utilisation plutôt large de la notion de risque par les acteurs sociaux et son caractère stratégique pour la mise en évidence de certaines préoccupations dans le cadre du débat public mais également vis-à-vis des autorités de régulation (Section 1) l'acception beaucoup plus restrictive de cette notion par les autorités de régulation - définissant le champ des risques reconnus - a été examinée (Section 2). Nous allons à présent tenter de passer en revue les principaux arguments à l'appui des ces deux positions divergentes, en commençant par celle défendue par les instances de régulation.

Nous avons vu précédemment que le champ des risques reconnus par les instances de régulation - système d'évaluation et gestion des risques classique - est délimité d'un côté par la définition des objets valorisés par la société et de l'autre par la seule méthode reconnue comme capable d'identifier et de valider un risque - la méthode scientifique. Le principal argument sur lequel se fonde cette approche à trait aux attributs de la méthode scientifique et en particulier à sa capacité à établir des faits avec certitude. La méthode scientifique serait donc la seule qui permette de distinguer entre l'arbitraire et le justifié, entre le risque qu'il faut prévenir et celui qui peut être ignoré car infondé. C'est donc la capacité de fournir des preuves quant à l'existence d'un risque qui constitue le point déterminant. Examiner cet argument de manière générale nous porterait bien au-delà du cadre de cet article, c'est pourquoi nous nous bornerons à questionner son bien-fondé dans le cas précis et délimité des organismes génétiquement modifiés disséminés dans l'environnement.

${ }_{37}$ Voir dans cet ouvrage l'article de Mark Hunyadi, «Le raisonnement du principe de précaution ». 


\subsubsection{Quel est le degré de preuve auquel peut arriver la science en matière de risques liés aux disséminations d'OGM?}

De manière générale, l'existence d'un risque est démontrée par l'établissement d'un lien entre une cause et un effet (dommage). Concrètement, les disciplines scientifiques qui permettent de prouver ou de formuler l'hypothèse de ce lien causal utilisent différentes méthodes:

- Expérimentale in vitro: Ayant pour cadre l'espace confiné d'un laboratoire, cette méthode s'applique à des systèmes simples, sur une période de temps généralement brève et dont le nombre de variables est limité et contrôlé. Si un lien causal peut être démontré, ce résultat fait office de preuve quant à ce qui peut survenir in vitro. En revanche, l'extrapolation de ce résultat à des conditions in vivo ne peut prétendre à d'autre statut que celui d'hypothèse, plus ou moins fondée selon les cas.

- Expérimentale in vivo: Cette méthode est évidemment celle qui, de la manière la plus directe, permet d'établir l'existence d'un risque. Toutefois, la complexité souvent extrême des systèmes in vivo pose des difficultés telles que l'existence d'un lien causal ne peut être déterminé que dans un nombre restreint de cas, lorsque la cause et l'effet sont peu distants à la fois dans le temps et dans l'espace et lorsque les interactions avec d'autres paramètres du système sont réduites au minimum.

- Statistique $^{38}$ : Contrairement aux méthodes expérimentales in vitro et in vivo, de même qu'à la modélisation informatique, la statistique se base sur une approche rétrospective du risque. L'expérience doit être réalisée un grand nombre de fois pour qu'une analyse statistique puisse être effectuée et qu'une corrélation entre une cause et un effet puisse être établie. Cette caractéristique fait de la statistique une méthode peu adaptée aux risques nouveaux et potentiellement graves associés aux OGM et de manière générale à nombre de nouvelles technologies. Par ailleurs, un présupposé majeur de la statistique, celui qui précisément permet à cette méthode de formuler des prédictions pour l'avenir, est celui de la constance des paramètres influençant le risque. Ce présupposé a acquis un statut de quasi-vérité dans de nombreux domaines comme par exemple celui des accidents de la route. Toutefois, au vu du caractère éminemment dynamique et évolutif des écosystèmes et des populations qui les composent de même qu'en regard de la connaissance limitée dont nous disposons, supposer une telle constance des paramètres semble être une hypothèse peu adaptée au cas de disséminations d'OGM.

\footnotetext{
Il convient de noter que des incertitudes statistiques sont présentes dans chacune des quatre méthodes décrites ici, et non pas seulement dans celle définie comme «statistique». Toutefois, une différence sépare les méthodes expérimentales in vitro et in vivo des méthodes dites statistiques et de modélisation informatique. En effet, les méthodes in vitro et in vivo ne sont applicables que lorsque la probabilité d'occurrence d'un risque est assez grande pour être déterminée expérimentalement. Les deux autres méthodes, dites statistiques et de modélisation informatique, doivent être utilisées au contraire lorsque cette probabilité est faible, car on doit alors disposer d'un échantillon suffisamment grand pour déterminer la probabilité de manière fiable.
} 
- Modélisation informatique: Cette méthode, qui est avant tout prospective, a été conçue dans le but d'appréhender des situations caractérisées par un haut degré de complexité. La modélisation informatique permet ainsi d'intégrer un nombre important de variables et, à travers une simulation, de reproduire des conditions in vivo. Le laps de temps considéré peut être également étendu de manière à envisager les effets à long terme. Toutefois, le recours à la modélisation informatique est difficilement capable de fournir des résultats qui feront office de preuve quant à l'existence d'un risque. Par contre, cette méthode peut être d'une grande utilité dans le cadre de l'application du principe de précaution en vue de développer des hypothèses avec une certaine solidité.

Evaluer les risques des OGM une fois mis en circulation dans un système ouvert, c'est-à-dire une fois disséminés dans l'environnement, cultivés à grande échelle, commercialisés, consommés par des êtres humains et des animaux, transportés d'un continent à l'autre dans des écosystèmes très divers résulte d'une tâche des plus ardues. En plus d'être insérés dans des systèmes ouverts caractérisés par de multiples incertitudes, les organismes transgéniques ont également, en commun avec l'ensemble des êtres vivants, la capacité de se reproduire, d'interagir avec leur environnement, de s'adapter à celui-ci et d'évoluer.

La force de l'expérimentation in vitro tient en sa très haute capacité d'analyse résultant de la simplification d'une situation complexe à un modèle expérimental dans lequel les interférences ont été éliminées et dont les variables sont à la fois limitées en nombre et contrôlées au niveau de leur variation. Une relation de cause à effet qui, dans un environnement complexe serait impossible à identifier peut ainsi être mise en évidence par de telles expériences. Toutefois, l'extrapolation des résultats obtenus in vitro à des situations in vivo, bien que très utile et fort courante, ne peut prétendre à un autre statut que celui d'hypothèse. L'exemple des risques de transfert horizontal d'ADN est à ce titre une bonne illustration des limites inhérentes aux expérimentations in vitro pour prouver l'existence de risques liés aux OGM. Il a été démontré qu'un transfert horizontal d'ADN provenant d'une plante transgénique à des bactéries du sol peut avoir lieu dans des conditions in vitro optimales, mais à des fréquences très faibles ${ }^{39}$. Ce résultat, qui peut être considéré comme une preuve quant à l'occurrence de ce transfert horizontal dans un environnement confiné présentant des conditions optimales ${ }^{40}$, a

39 Schluter K., Futterer J. and Potrykus I. (1995), «Horizontal gene transfer from a transgenic potato line to a bacterial pathogen (Erwinia chrysanthemi) occurs - if at all - at an extremely low frequency », Biotechnology (N Y), Oct, Vol. 13, n 10, pp. 1094-1098.

Gebhard F. and Smalla K. (1998), «Transformation of Acinetobacter sp. Strain BD413 by Transgenic Sugar Beet DNA», Applied and Environmental Microbiology, Vol. 64, n 3, pp. 1550-1554.

De Vries J., Meier P. and Wackernagel W. (2001), «The natural transformation of the soil bacteria Pseudomonas stutzeri and Acinetobacter sp. by transgenic plant DNA depends strictly on homologous sequences in the recipient cells», FEMS Microbiology Letters Vol. 195, pp. 211-215.

40 Les expériences effectuées dans des conditions in vivo n'ont jamais permis de mettre en évidence un transfert horizontal d'ADN de plante à une bactérie du sol. Aucune conclusion ne peut donc être tirée de tels résultats ou plutôt d'une telle absence de résultats. Absence d'évidence ne signifiant pas évidence d'absence, un tel résultat négatif ne peut en aucun cas avoir valeur de preuve. A moins d'avoir analysé de manière systématique la diversité des écosystèmes, leurs variations saisonnières, etc. l'absence de résultat peut simplement signifier que l'on n'a pas cherché au bon 
ensuite été extrapolé à des conditions in vivo, donnant lieu à des conclusions radicalement différentes. Certains auteurs ont ainsi conclu à une probabilité de transfert horizontal insignifiante dans le cas de disséminations d'OGM ${ }^{41}$ alors que d'autres auteurs ont interprété ces résultats comme une indication que de tels transferts risquaient effectivement d'avoir lieu ${ }^{42}$. Personne ne s'y trompera, une telle variabilité des interprétations tient au fait que le domaine de la preuve a été définitivement quitté pour laisser place à celui de l'hypothèse, les caractéristiques et la variabilité des conditions in vivo ne pouvant être établies que sur la base de présupposés plus ou moins fondés. Cet exemple illustre ainsi clairement les limites de l'expérimentation in vitro pour l'établissement de preuves quant à l'existence ou à l'absence de risques dans un environnement ouvert.

En ce qui concerne les dispositifs expérimentaux en plein champ ou in vivo, il a été remarqué précédemment que leur caractère opérationnel est limité à des risques résultant d'une relation causale simple et limitée dans le temps et l'espace, dans laquelle peu de paramètres extérieurs viennent interférer. Autrement dit, lorsqu'une situation réelle s'apparente, du point de vue de la complexité, à une situation in vitro, alors les expériences en plein champ permettent d'établir des preuves quant à l'existence d'un risque. Un lien de causalité entre la dissémination du pollen de colza transgénique et son hybridation subséquente avec des plantes sauvages apparentées a pu ainsi être démontré par des expériences in $v_{i v o}{ }^{43}$. En revanche, étudier l'impact du pollen transgénique sur certains prédateurs suite à son passage à travers une chaîne trophique complexe est susceptible de se révéler une tâche d'une complexité insondable, en l'état actuel des connaissances et avec les moyens techniques dont nous disposons.

Mais, au-delà de la difficulté de la tâche, reste la question de l'opportunité d'effectuer des tests grandeur nature lorsqu'il s'agit de démontrer l'existence de risques bien souvent caractérisés par leur haut degré de gravité et d'irréversibilité. A noter à ce sujet que des expériences portant sur un lien causal complexe, dont les dimensions spatiales et temporelles sont considérables ont plus de chances d'impliquer des dommages graves et irréversibles que des expériences de dimension réduite, portant sur une saison et n'impliquant qu'un contact très restreint avec le reste de l'écosystème (filet à pollen et à insectes, isolation du sol, élimination des plantes en fin de saison, stérilisation du sol, etc.). La gravité et l'irréversibilité des dommages qui pourraient résulter de test in vivo ne peut donc être considérée comme une constante, mais plutôt comme proportionnelle aux dimensions spatio-temporelles du test. Les risques associés à une dissémination expérimentale de petite taille ne sont donc en aucun cas comparables à ceux résultant d'une dissémination commerciale à grande échelle dépourvue d'encadrement et de suivi.

Ainsi, malgré sa contribution essentielle à la connaissance des risques liés aux OGM dans un environnement ouvert, l'expérimentation in vivo est limitée tant du

endroit, ou encore que la méthode et l'instrumentation utilisée n'étaient pas suffisamment sensible ou inadaptées.

${ }^{41}$ Schluter K., Futterer J. and Potrykus I. (1995), op. cit.

42 Ho M-W., «Horizontal Gene Transfer - The Hidden Hazards of Genetic Engineering », Institute of Science in Society, Url: http://www.i-sis.org.uk/horizontal.php

43 Chèvre A-M., Eber F. and Renard M. (1998), op. cit. 
point de vue des moyens dont elle dispose pour appréhender une réalité complexe que du point de vue des conséquences potentiellement dommageables des expérimentations elles-mêmes.

Face à de telles limitations, le recours à des systèmes de modélisation permet de prendre en compte les nombreuses variables présentes dans un système ouvert, les interactions de ces variables entre elles et leur évolution «concertée » dans le temps. La modélisation informatique peut s'avérer donc très utile pour formuler des hypothèses et en vérifier la solidité, mais les résultats obtenus dans ce cadre ne pourront que difficilement faire office de preuve quant à l'existence d'un lien causal.

En conclusion, lorsque l'on a affaire à des systèmes ouverts tels que celui des organismes génétiquement modifiés disséminés dans l'environnement, l'établissement de preuves quant à l'existence de risques faisant intervenir des interactions complexes, sur le long terme et sur des territoires étendus s'avère à la fois difficile et parfois non souhaitable au vu des dommages qu'une telle preuve serait susceptible d'occasionner. La preuve ayant largement cédé la place à l'hypothèse, on peut se demander si le recours à la précaution n'est pas destiné à devenir la règle et la prévention l'exception, contrairement à ce qui a été envisagé jusqu'à présent. La précaution est en effet considérée par de nombreux instruments juridiques comme une étape transitoire, ne perdurant que jusqu'au moment où les connaissances scientifiques permettront d'établir avec certitude l'existence d'un risque, induisant ainsi la transition de la précaution à la prévention ${ }^{44}$. Or, dans le domaine environnemental notamment, mais également dans celui de la santé, les risques à long terme ainsi que ceux issus d'interactions complexes sont typiquement des risques au sujet desquels les incertitudes ne seront que difficilement réduites. Dans de tels cas, la précaution ne pourra donc pas être appliquée de manière transitoire, mais il faudra au contraire s'attendre à ce qu'elle perdure sur le long terme.

En examinant les «preuves» qui peuvent en définitive être fournies par les disciplines scientifiques - celles faisant partie de la science reconnue par les instances de régulation - on arrive à la conclusion que, hors du laboratoire, en situation in vivo, la contingence et la multitude des paramètres ne permet que difficilement à la méthode scientifique de formuler plus qu'une hypothèse bien fondée. Dès lors, l'argument selon lequel seule la méthode scientifique permet de sortir de l'arbitraire en déterminant quels sont les risques pertinents et objectifs et quels sont ceux qui ne le sont pas, est caduc. Nous avons en effet montré que la capacité à fournir des certitudes, qui est attribuée à la science par les instances de régulation, semble devoir être relativisée au point de voir la précaution devenir le cadre général dans lequel les risques relatifs au développement des biotechnologies peuvent être appréhendés. Il en résulte alors que plus rien ne justifie - en tous cas dans ce cadre précis - le statut conféré à la science qui seule pourrait déterminer quels risques méritent d'être pris en compte et quels risques doivent être seulement considérés comme des menaces.

${ }^{44}$ Voir en particulier l'art. 5 al. 7 de l'accord sur l'application des mesures sanitaires et phytosanitaires (SPS) de l'OMC. 


\subsection{Quelles justifications pour une acception élargie des risques reconnus?}

Mais passons à présent au point de vue des acteurs sociaux. Leur approche impliquant un élargissement du champ des risques reconnus est basée sur des nécessités multiples dont le point commun semble être le besoin d'un meilleur contrôle démocratique sur le développement des biotechnologies.

\subsubsection{Besoin d'un meilleur contrôle démocratique sur le développement des biotechnologies}

Un constat fort simple est à l'origine du point de vue développé par les acteurs sociaux. Il s'agit du fait que tout développement technologique a des répercussions à de multiples niveaux et pas uniquement sur le plan technique ou scientifique. Bien qu'évident et documenté de manière empirique, cet argument est pourtant souvent ignoré. Or, ayant démontré le caractère injustifié du recours exclusif à la méthode scientifique pour valider un risque, l'existence même de répercussions sur le plan socio-économique ou éthique ne devrait-elle pas suffire à justifier le point de vue des acteurs sociaux? Nos sociétés démocratiques peuvent-elles encore se soustraire à la demande sociale d'élargissement du champ des risques reconnus aux risques éthiques, économiques ou sociaux - dont la controverse publique sur les OGM peut être considérée comme l'expression?

Si l'on admet la pertinence de ce questionnement, alors force est de convenir que le champ des risques à prendre en compte devrait être défini en premier lieu par la société, au travers d'un processus permettant la mise en évidence des objets valorisés ${ }^{45}$. Il en découle que les risques reconnus ne devraient donc pas être déterminés par le champ d'application de la science, mais que leur identification devrait être subordonnée à la définition des objets valorisés par la société, quels qu'ils soient.

Reste la question complexe de savoir comment faire - par quelle méthode, selon quelle procédure - pour identifier et valider des risques de nature sociale, économique ou éthique. Au caractère forcément à la fois inventif, novateur et complexe de telles procédures, la théorie de la science politique n'a été que de peu d'utilité et c'est avant tout dans la pratique que des éléments de réponse à cette question ont pu être trouvés. A travers ses nombreuses analyses des controverses publiques relatives aux risques technologiques, la sociologie des sciences a ainsi mis en évidence nombre de situation dans lesquelles les «débordements», à savoir les effets inattendus (qui peuvent constituer autant de risques), à la fois techniques et sociaux, résultant du développement des sciences et des techniques, ont été identifiés, débattus et redéfinis. Les espaces publics dans lesquels ces controverses se développent ont été définis comme des forums hybrides. «Forum » parce qu'il s'agit d'espaces ouverts où des groupes peuvent se mobiliser pour débattre de choix techniques qui engagent le collectif. Hybrides, parce que ces groupes engagés et les porte-parole qui prétendent les représenter sont hétérogènes: on y trouve à la fois des experts, des hommes politiques, des techniciens et

Comme mentionné précédemment, nombre d'objets valorisés par la société ont trait aux conditions économiques ou sociales, de même qu'à des conditions d'existences jugées moralement préférable. 
des profanes qui s'estiment concernés. Hybride, également, parce que les questions abordées et les problèmes soulevés s'inscrivent dans des registres variés, qui vont de l'éthique à l'économie en passant par la physiologie, la physique atomique et l'électromagnétisme ${ }^{46}{ }^{47}$. Mais, sans règles du jeu, le développement des forums hybrides a également eu des effets contreproductifs en ne répondant qu'à la logique des rapports de force entre les acteurs, ou alors en donnant lieu à des utilisations contraires à leur propos initial qui est de favoriser la démocratisation des choix technologiques ${ }^{48}$. Afin de pallier ces possibles travers, des procédures plus organisées ont vu le jour. Connue sous le nom générique de participative Technology Assessment (pTA), cette grande famille regroupe des procédures aux caractéristiques variables telles que les «focus groups», les «conférences de consensus» (en France) ou «Publiforums» (en Suisse) ${ }^{49}$.

Associés à des recherches dans tous les domaines pertinents, les forums hybrides et les pTA sont donc bien susceptibles, selon nous, de faire office de procédures à travers lesquelles des risques de nature sociale, économique ou éthique peuvent être identifiés et validés. Or, malgré leur nombre croissant, la question de la prise en compte par les instances de décision des résultats de ces procédures participatives reste encore problématique. Il en découle que, dans bien des cas, la situation au niveau de la prise de décision n'a que peu changéso. Plutôt que de fonder l'édifice permettant de contrôler et d'orienter le développement des biotechnologies sur une définition des objets valorisés et des risques y relatifs qui ait fait l'objet d'un débat et d'une négociation, c'est la définition plus ou moins arbitraire et opaque de ce qui fait partie de la science et de ce qui n'en fait pas partie qui limite de manière peu justifiée les risques permettant d'influer sur les développements de cette technologie.

Considérons par exemple un objet valorisé tel que le fait de disposer d'une alimentation saine et suffisante. La valeur accordée à cet objet se traduit notamment par le fait qu'il a été élevé au statut de droit universel pour toute l'espèce humaine $^{51}$. Malgré cela, un risque pour la sécurité alimentaire ne constitue pas une justification pour limiter le développement des biotechnologies. Or, comme il a été mis en évidence ${ }^{52}$, les biotechnologies sont susceptibles de causer un

46 La référence à ces disciplines scientifiques est due au fait que les auteurs ont pris les controverses relatives aux déchets radioactifs et aux lignes à haute tension comme exemple de forum hybrides.

Callon M., Lascoumes P., Barthes Y. (2001), op. cit., p. 34.

48 Callon M., Lascoumes P., Barthes Y. (2001), op. cit., pp. 210-215

49 Pour plus de détails, voir dans cet ouvrage: Kaufmann A., Perret H., Bordogna Petriccione B., Audétat M. et Joseph C., «De la gestion à la négociation des risques: apport des procédures participatives d'évaluation des choix technologiques.»

so Voir dans cet ouvrage: Perret H., Bordogna Petriccione B., Kaufmann A., Audétat M., Joseph C. «Disséminations expérimentales d'OGM en France et en Suisse: évolution du débat public ». Cet article examine, entre autres, cette question et montre que malgré certains changements, le poids de l'expertise scientifique (la prise en compte des risques sanitaires et environnementaux) reste dominant dans la prise de décision.

51 Le droit à l'alimentation est inscrit dans la déclaration universelle des droits de l'homme, art. 25, al.1: «Toute personne a droit à un niveau de vie suffisant pour assurer sa santé, son bien-être et ceux de sa famille, notamment pour l'alimentation, l'habillement, le logement, les soins médicaux ainsi que pour les services sociaux nécessaires; [...].»

s2 Voir notamment Cullet P. (2003) «Food security and intellectual property rights in developing countries », Les cahiers du RIBios n 6 , RIBios-IUED, Genève. 
dommage à cet objet valorisé: à travers le système des brevets, qui est peu adapté au type d'agriculture pratiqué dans de nombreux pays du Sud, les biotechnologies risquent d'augmenter la dépendance des paysans vis-à-vis de l'agro-industrie et résulter en une précarisation de leur condition susceptible d'affecter négativement leur accès à la nourriture. Malgré un certain nombre de données qui permettent de donner quelque crédit à l'existence d'un risque pour la sécurité alimentaire, ce dernier ne peut être pris en compte car il n'entre pas dans le cadrage des disciplines considérées comme scientifiques.

\subsubsection{Orienter le développement technologique dès le départ}

Mais outre la nécessité, directement exprimée par les acteurs sociaux, d'accroître le contrôle démocratique sur le développement des biotechnologies, d'autres arguments plaident en faveur de l'accession des menaces au statut de risques reconnus. Le premier concerne le caractère indispensable de la prise en compte des menaces pour pouvoir orienter le développement d'une technologie dès le départ (en amont) et non pas, comme c'est généralement le cas, lorsque la technologie a déjà été développée jusqu'au stade de mise sur le marché de divers produits (en aval). En effet, les risques reconnus par les instances de régulation sont des risques qui ne sont identifiables qu'à des stades avancés du développement d'une technologique. Ce n'est que lorsque cette dernière commence à prendre la forme de divers produits, applications ou utilisations qui vont s'insérer de manière tangible dans divers contextes que des risques reconnus peuvent être identifiés.

Cet aspect concret et contextuel des risques reconnus est une sérieuse limitation pour les acteurs qui, à travers la recherche de nouveaux risques, souhaitent motiver une action qui ne se borne pas à une stratégie d'évitement en aval mais qui permette également de penser et de questionner dès le départ un projet technologique tel que les OGM. Le fait de prendre en compte les menaces devrait donc permettre d'intervenir davantage en amont du développement technologique et de permettre ainsi d'envisager des développements technologiques alternatifs. La difficulté rencontrée pour formuler des alternatives à la technologie dominante serait donc directement liée au type de risques reconnus par les instances de régulation. Ce qui n'est considéré que comme des menaces correspond en effet le plus souvent à des risques de type socio-économique ou éthique qui viennent questionner l'ensemble du développement technologique, ses conséquences et les motivations qui ont présidé à son développement.

\subsubsection{Clarification du débat}

Le dernier point favorable à l'élargissement du champ des risques reconnus concerne la clarification du débat qui en résulterait. Comme déjà mentionné, les risques reconnus par les instances de régulation sont ceux qui tendent à être le plus fréquemment invoqués dans le débat public puisqu'ils sont les seuls à justifier des modifications, des ralentissements, voire des arrêts du développement technologique. Il en résulte que, alors même qu'elles sont souvent l'expression des motivations les plus profondes des acteurs sociaux, les menaces n'occupent qu'une place mineure dans le débat. 
Dans le cadre des demandes de dissémination expérimentales en Suisse par exemple, leur prise en compte aurait permis de débattre des véritables enjeux en présence et non pas uniquement des risques de dissémination du pollen ou des risques liés à la présence de gènes de résistance aux antibiotiques. Ces risques scientifiques touchant la santé et l'environnement constituant l'unique justification propre à limiter de telles expérimentations, ils ont été pratiquement les seuls à être invoqués et débattus dans le cadre de la procédure d'autorisation ${ }^{53}$. Or, dans de telles situations, il est difficile d'éviter une double utilisation des risques sanitaires et environnementaux: l'une, directe, visant uniquement l'évitement de ces risques et l'autre, indirecte, visant plutôt l'évitement des menaces non prises en compte dans la procédure d'autorisation. Un tel «usage indirect», s'il est compréhensible, est toutefois grandement préjudiciable à la clarté et à la franchise du débat public. L'accession des menaces au statut de risque reconnu permettrait probablement de résoudre ce problème qui constitue souvent un obstacle majeur au dialogue pourtant nécessaire entre la communauté scientifique, les instances de régulation et les acteurs sociaux.

\subsection{Elargissement du champ des risques reconnus et précaution}

Nous allons à présent tenter de faire la relation entre la question de l'élargissement du champ des risques reconnus et la notion de précaution. Il existe en effet entre les deux un lien qui mérite d'être explicité. Du fait de la complexité de la question et de son caractère encore très ouvert, nous nous limiterons toutefois ici à exposer certains éléments à titre de pistes de réflexion.

Nous avons vu précédemment que, en rendant légitime le fait de prendre des mesures d'évitement face à des risques qui ne peuvent être prouvés, la précaution a permis d'élargir le champ des risques reconnus par les instances de décision. Elle a permis de faire sortir certains risques du domaine des menaces, pour les faire entrer en pleine lumière dans celui des risques qui peuvent être validés et reconnus, tout en les baptisant d'un nouveau nom: les risques hypothétiques. En reconnaissant la limite inhérente à toute connaissance scientifique ainsi qu'à sa capacité de prévenir et de gérer les risques technologiques, la précaution a ébranlé la vision positiviste de la science. Comme le souligne Godard, «avec la précaution, c'est d'abord une prise de distance vis-à-vis de la science et de la technique qui se manifeste, comme en écho au désenchantement de l'opinion publique à leur endroit $»^{54}$.

Cette prise de distance de la précaution vis-à-vis de la science est toutefois limitée. La prévention se distingue de la précaution en ce sens que, pour la première, le lien de causalité existant entre un dommage éventuel et sa cause a pu être établi de manière certaine alors que pour la seconde, cette causalité n'a pu être vérifiée et elle fait donc l'objet d'hypothèses plus ou moins fondées ${ }^{55}$. Les risques avérés - dans le cadre de la prévention - tout comme les risques hypothétiques dans le cadre de la précaution - ont respectivement été prouvés ou ont fait l'objet

\footnotetext{
Voir dans cet ouvrage: Perret H., Bordogna Petriccione B., Kaufmann A., Audétat M., Joseph C. «Disséminations expérimentales d'OGM en France et en Suisse: évolution du débat public»

s4 Godard O. (1997), op. cit., p. 38.

s5 Voir dans cet ouvrage: Hunyadi M., «La logique du raisonnement de précaution».
} 
d'hypothèses selon la méthode de validation reconnue qui est la méthode scientifique. Ainsi, le continuum qui va du risque avéré au risque hypothétique faiblement plausible en passant par un risque hypothétique fondé sur une hypothèse solide est directement proportionnel à la valeur justificative du risque pour la prise de mesure ${ }^{56}$. La précaution autorise en effet la prise de mesures même si l'on ne dispose que d'une hypothèse. Or, cette hypothèse doit être fondée scientifiquement. De plus lorsqu'il s'agit de prouver cette hypothèse, seule la science demeure valable ${ }^{57}$.

Ainsi, force est de constater que, malgré l'élargissement consenti à travers l'adoption de la précaution, seule une minorité des risques nombreux et fort divers qui apparaissent dans le débat public entrent dans le spectre des risques qui peuvent être validés et reconnus. Car si la précaution a élevé au statut de risque reconnu ces menaces qui entrent dans le cadrage des disciplines scientifiques mais que ces dernières n'arrivent pas encore à prouver, elle n'a en revanche pas remis en question le bien-fondé de ce cadrage. Bien qu'ayant permis une prise de distance par rapport à la science en en reconnaissant les limites, la précaution ne s'est pas complètement affranchie de l'autorité exclusive accordée à la science pour définir le risque.

L'avènement de la précaution semble donc laisser entière une question primordiale: Que dire et que faire de toutes les menaces condamnées à une non-existence certaine en regard du processus d'évaluation des risques? La précaution n'auraitelle aucun rôle à jouer à leur sujet?

Que ce soit d'un point de vue théorique ou d'un point de vue pratique, la précaution ne s'est pas encore stabilisée dans une forme définitive. Diverses écoles, diverses pratiques, mais également diverses revendications s'affrontent ou se complètent. Dans ce foisonnement, on distingue toutefois une tendance allant dans le sens d'un élargissement du rôle de la précaution à tous les types de menaces découlant du développement d'une technologie.

Les tenants de ce qui a été défini ailleurs dans cet ouvrage comme l'«école dialogique » de la précaution ${ }^{58}$ sont certainement parmi les représentants les plus radicaux de cette tendance. Lorsque, à travers divers exemples, les auteurs de «Agir dans un monde incertain ${ }^{59}$ examinent les controverses suscitées par divers développements technologiques (OGM, nucléaire) et les incertitudes qui les accompagnent, ils montrent bien que «les controverses engendrées par ces incertitudes vont bien au-delà des seules questions techniques $»^{60}$. La reconnaissance du fait que les développements technologiques ont des répercussions non seulement d'un point de vue technique, mais également social, et que ces répercussions

56 Voir à ce sujet un graphique très explicite dans: Godard O., «De nouvelles donnes, une nécessaire nouvelle approche», Maîtrise des risques - Prévention et principe de précaution, Actes du Colloque du 6 novembre 2001, Institut Pasteur, pp. 69-72.

57 Voir Christoforou T. (2002), «Science, law and precaution in dispute resolution on health and environmental protection: what role for scientific experts?», in Le commerce international des organismes génétiquement modifiés, éd. La documentation française, Paris, pp. 221-222.

58 Voir dans cet ouvrage l'article de Hunyadi M. «le raisonnement de précaution».

59 Callon M., Lascoumes P., Barthes Y. (2001), op. cit.

6o Callon M., Lascoumes P., Barthes Y. (2001), op. cit., p. 45. 
engendrent des incertitudes qui peuvent être autant de menaces ou de risques, a induit les auteurs à qualifier ce type de controverse de «socio-technique».

L' «école dialogique» remet en question de manière radicale l'approche technicienne qui caractérise la gestion des risques technologiques. Ainsi, selon Roy, «alors que la conception rationnelle-légale wébérienne de la conduite des affaires publiques s'appuie sur l'idée que le bien-fondé d'une décision dépend d'une bonne caractérisation scientifique du problème à traiter, les politiques procédurales $^{61}$ puisent quant à elles leur légitimité sur la mise en place d'espaces collectifs où sont négociées les conséquences socio-économiques et politiques de l'introduction d'une technologie [...] Cette tendance à la diversification des espaces de négociation en amont de la décision à laquelle nous assistons en France peut être appréhendée dans la continuité de l'attitude critique et réflexive qui est contenue dans le principe de précaution vis-à-vis du rôle central de l'expertise scientifique dans l'encadrement des risques techniques.» ${ }^{62}$

De par son approche sociologique, l'école dialogique a le mérite d'avoir su écouter les préoccupations des acteurs de la société civile et d'avoir conféré à la précaution une dimension qui soit à la hauteur des problèmes soulevés. Comme le souligne Roy, «la mobilisation du principe de précaution par différents acteurs comme Greenpeace, la Confédération paysanne et les politiques peut ainsi apparaître comme une solution alternative aux lacunes dénoncées sur le fonctionnement de la CGB [Commission génie biomoléculaire] en particulier et sur les modalités de prise de décision en général.» ${ }^{63}$ Ainsi, « on peut analyser la controverse actuelle comme une critique de cette conception de l'expertise privilégiant le savoir scientifique au détriment de savoirs portant sur les dimensions sociales et économiques induites par l'introduction des OGM ${ }^{64}$. La prise en compte des dimensions sociales, économiques, mais également éthiques et politiques relatives aux OGM est demandée à grande voix par toutes sortes d'acteurs sociaux.

La position de la Commission française du développement durable (CFDD) est à ce titre une bonne illustration de la teneur de la demande sociale au sujet du principe de précaution. En donnant son avis concernant le rapport Kourilsky \& Viney sur le principe de précaution, la CFDD remarque en effet: «[...] Le choix d'une approche limitée aux risques sanitaires et environnementaux conduit à confiner l'application du principe de précaution, en omettant de l'appliquer aux conséquences premières de l'innovation technique sur la société, aussi bien en termes économiques qu'en termes de développement, d'emploi, d'équité sociale, de solidarité Nord-Sud, etc. L'exigence du développement durable et la pratique qui se dégage des expériences récentes conduisent en effet à élargir la notion de principe de précaution bien au-delà des risques sanitaires et environnementaux, par exemple jusqu'aux différents problèmes de société qu'induisent les innovations technologiques.» ${ }^{65}$

${ }^{61}$ La mise en place de procédures capables de contribuer à la démocratie dialogique constitue l'un des outils principaux de l'école dialogique (note de l'auteur).

62 Roy A. (2001) «Les experts face au risque: le cas des plantes transgéniques », Presse universitaire de France, coll. Partage du savoir, Paris, p. 263.

63 Roy A., op. cit., p. 255.

${ }^{64}$ Roy A., op. cit., p. 249.

65 Testart J. (2003) «Réflexions pour un monde viable, propositions de la commission française du développement durable (2000-2003)», éd. Mille et une nuits, pp. 24-25. 
Il semble donc que l'évitement des risques scientifiques ne soit pas suffisant à régler les problèmes posés par une nouvelle technologie telle que les OGM. Toujours selon Roy, «au-delà de l'impératif de réduction des risques, l'enjeu du débat porte sur l'explication et la négociation des "conséquences politiques et sociales du développement technologique et d'introduction de cette dimension dans le débat politique pour en permettre une maîtrise sociale accrue" $\gg^{66}$.

Avec une compréhension du problème peut-être quelque peu différente de celle des précédents auteurs («école dialogique»), le fait de considérer la précaution comme le biais par lequel la politique peut refaire son entrée au niveau de la gestion des développements technologiques sous-entend également - même si dans une mesure moindre - la possibilité de prendre en compte des dimensions qui ne sont pas strictement scientifiques ${ }^{67}$. Selon Godard, la précaution réintroduirait ainsi une certaine distance entre science et décision publique et elle permettrait de même de «restaurer la primauté du politique dans la définition des problèmes et l'évaluation de l'opportunité de l'engagement d'une action publique $»^{68}$. Bien qu'il permette de restaurer une certaine distinction entre la sphère de l'expertise et celle de la décision ${ }^{69}$, entre l'évaluation des risques et la gestion des risques, ce retour du politique a en réalité une portée assez limitée. La Communication de la Commission européenne note ainsi: «Le choix de la réponse à donner face à une certaine situation résulte dès lors d'une décision éminemment politique, fonction du niveau de risque "acceptable" par la société devant supporter le risque ${ }^{70}$. C'est donc sur le degré d'acceptabilité des risques préalablement identifiés et évalués pat les experts scientifiques que porte la latitude du politique, et non pas sur le type de risque à prendre en compte.

Tout comme le concept de précaution lui-même, la nature du lien entre élargissement des risques reconnus et précaution est une question encore ouverte. Un rapide survol des arguments en faveur et en défaveur d'une application de la précaution à tous les types de risques montre d'ailleurs qu'il s'agit d'une question difficile à trancher. Sans prétendre à une quelconque exhaustivité, on peut mentionner le fait qu'un tel élargissement de la portée de la précaution, en provoquant un éloignement de son cadre d'application originel - celui des risques scientifiques, qu'ils soient environnementaux ou sanitaires - est susceptible d'aboutir à une dénaturation et à une dilution du concept ainsi qu'à une diminution de sa portée. Il serait en outre intéressant d'examiner dans quelle mesure d'autres principes ne pourraient pas avantageusement remplir ce rôle de formulation d'une exigence de prise en compte des risques «non scientifiques». D'un autre côté en

66 Roy A., op. cit., p. 253, (Moatti J-P., Lochard J. (1987) cités par Roy)

${ }_{67}$ Cette position, dans la mesure où elle se réfère à la prise en charge par les politiques des menaces sociales, politiques ou éthiques relatives à une technologies, ne correspond vraisemblablement pas à celle défendue par les tenants de l'école dialogique puisque ces derniers voient justement dans la double délégation - aux experts scientifiques d'une part et aux politiciens d'autre part l'origine du déficit démocratique qui caractérise la gestion des nouvelles technologies.

68 Godard O. (1997), op. cit., p. 65.

69 La décision prise en février 1997 par le Premier Ministre français Alain Juppé de ne pas autoriser la culture de semences de maïs Bt de Novartis est un bon exemple d'une telle prise de distance des politiques vis-à-vis de l'expertise scientifique.

70 Commission de la Communauté Européenne (2000), op. cit., p. 16. 
revanche, une telle acception de la portée de la précaution présente l'avantage d'être assez proche de la demande sociale relative à ce concept. De plus, en étant déjà largement popularisée et inscrite dans de nombreux textes de lois, la seule extension de la portée de la précaution permettrait un changement plus rapide que si un nouveau concept devait être avalisé.

\section{CONCLUSION}

Après avoir développé l'approche des acteurs sociaux visant à mettre au même niveau et donc à faire reconnaître tous les types de risques, qu'ils soient sanitaires, environnementaux, économiques, éthiques ou sociaux, nous avons examiné l'approche des instances de régulation qui se base sur une distinction claire entre les risques reconnus - les risques sanitaires et environnementaux - et les risques non reconnus.

En comparant ces deux approches, il apparaît que, dans le cas des OGM pour le moins, la pratique actuelle - celle des instances de régulation - ne se justifie que très difficilement face aux arguments divers sur lesquels se fonde la demande d'élargissement des risques reconnus.

Dans le cas des OGM, les limites de la science pour fournir une preuve quant à l'existence d'un risque sont telles qu'il semble difficilement soutenable de conférer aux disciplines scientifiques le statut d'unique méthode capable de distinguer l'arbitraire du justifié. Dès lors, il ne semble pas raisonnable d'évaluer les risques scientifiquement démontrables tout en ignorant ce qui n'est qu'impact, question, problème et qui relève du social, de l'économique, de l'éthique ou du culturel.

La reconnaissance de toutes les menaces aurait par ailleurs l'inestimable avantage de permettre une clarification des arguments de la controverse en rendant possible le débat sur tous les enjeux en présence.

Mais le plus intéressant reste tout de même le fait que l'accession des menaces au statut de risque reconnu serait susceptible de permettre un retournement de situation pour le moins inattendu. S'il est vrai que penser une situation en termes de risques implique divers désavantages au premier rang desquels le fait qu'il s'agit d'une approche en réaction, souvent négative et contestataire, cela permet toutefois de prendre en compte des menaces intervenant en amont du développement technologique, rendant ainsi possible la discussion et la formulation de proposition de développement technologiques alternatifs. Un changement radical de perspective pourrait dès lors s'opérer avec l'adoption d'une approche positive, voire proactive de «mise en alternative» des diverses possibilités de développement technologique.

En conclusion, dans la mesure où la revendication des acteurs sociaux peut être considérée comme justifiée, l'utilisation élargie de la notion de risque, de par sa portée stratégique, constitue un outil dont l'utilité méritait d'être soulignée. 


\section{BIBLIOGRAPHIE}

Bonneuil C. (2002), «La question des cadrages de l'expertise en situation d'incertitude», Maîtrise des risques - Prévention et principe de précaution, Actes du Colloque du 6 novembre 2001, Institut Pasteur, pp. 81-94.

Butler D. and Reichhardt T. (1999), «Long-term effect of GM crops serves up food for thought», Nature, Vol. 398, pp.651-653

Callon M., Lascoumes P., Barthes Y. (2001), Agir dans un monde incertain, essai sur la démocratie technique, Seuil.

CFSB (2001), «Prise de position de la CFSB concernant la demande B00003 de l'Institut de biologie végétale de 1'EPFZ. Comportement des variétés de blé transgénique KP4 en plein champ.», url: http://www.buwal.ch/stobobio/cfsb/pdf/Prise_de_pos.pdf (accédé le 20 juin 2003).

Chateauraynaud F., Torny D. (1999), «Les sombres précurseurs: une sociologie pragmatique de l'alerte et du risque», Paris, éd. De l'Ecole de Hautes Etudes en sciences sociales, p. 80.

Chèvre A-M., Eber F. and Renard M. (1998), «Plantes génétiquement transformées: quels risques de dissémination de gènes dans l'environnement?», in Organisme Génétiquement Modifié à l' INRA, Environnement, Agriculture et Alimentation, INRA. Url: http://www.inra.fr/Internet/ Directions/DIC/ACTUALITES/DOSSIERS/OGM/OGM.htm

Claude Gilbert dans «La fin des risques?», in Quaderni, La revue de la communication, $\mathrm{n}^{\circ}$ 48, 2002, pp.111-120.

Commission des Communautés Européennes (2000), Communication de la Commission sur le recours au principe de précaution, Bruxelles, Url: http://europa.eu.int/eur-lex/fr/com/cnc/ 2000/com2000_0001fr01.pdf, pp. 18-19.

Christoforou T. (2002), «Science, law and precaution in dispute resolution on health and environmental protection: what role for scientific experts?», in Le commerce international des organismes génétiquement modifiés, éd. La documentation française, Paris, pp. 221-222.

Cullet P. (2003), Food security and intellectual property rights in developing countries, Les cahiers du RIBios $n^{\circ} 6$, RIBios-IUED, Genève.

De Vries J., Meier P. and Wackernagel W. (2001), «The natural transformation of the soil bacteria Pseudomonas stutzeri and Acinetobacter sp. by transgenic plant DNA depends strictly on homologous sequences in the recipient cells », FEMS Microbiology Letters Vol. 195, pp. 211-215.

Gebhard F. and Smalla K. (1998), «Transformation of Acinetobacter sp. Strain BD413 by Transgenic Sugar Beet DNA», Applied and Environmental Microbiology, Vol. 64, n 3, pp. 1550-1554.

Godard O.(1997), Le principe de précaution dans la conduite des affaires humaines, INRA, Paris

Godard O., De nouvelles donnes, une nécessaire nouvelle approche, Maîtrise des risques - Prévention et principe de précaution, Actes du Colloque du 6 novembre 2001, Institut Pasteur

Hellmich R. L. and Siegfried B. D. (2001), in Genetically Modified Organisms in Agriculture-Economics and Politics, ed. Nelson, G. C. (Academic, London), pp. 283-289.

Ho M-W., Horizontal Gene Transfer - The Hidden Hazards of Genetic Engineering, Institute of Science in Society, Url: http://www.i-sis.org.uk/horizontal.php

Howard T. et Rifkin J. (1977), Who Should Play God? The Artificial Creation of Life and What It Means to the Future of the Human Race?, Dell Publishing, New York.

James C. (1997), Global status of transgenic crops in 1997, ISAAA Briefs No.5, ISAAA:Ithaca, NY

Krimsky S. (1992), «Recombinant DNA research», in Controversy, Politics of technical decisions, Third Edition, Dorothy Nelkin, editor, Sage Publications, USA.

Pusztai A. (2002), «GM food safety: scientific and institutional issues», Science as Culture, Vol. 11, pp. 69-92.

Roy A. (2001), Les experts face au risque: le cas des plantes transgéniques, Presse universitaire de France, coll. Partage du savoir, Paris 
Schluter K., Futterer J. and Potrykus I. (1995), «Horizontal gene transfer from a transgenic potato line to a bacterial pathogen (Erwinia chrysanthemi) occurs - if at all - at an extremely low frequency », Biotechnology (N Y), Oct, Vol. 13, n 10, pp. 1094-1098.

Stanley-Horn D. E., Dively G. P., Hellmich R. L., Heather R. M., Sears M. K., Rose R., Jesse L.C., Losey J. E., Obrycki J. J. and Lewis L. (2001), «Assessing the impact of Cry1Ab-expressing corn pollen on monarch butterfly larvae in field studies» Proc. Natl. Acad. Sci., Vol. 98, pp.1193111936.

Testart J. (2003), Réflexions pour un monde viable, propositions de la commission française du développement durable (2000-2003), éd. Mille et une nuits.

van Zwanenberg P. et Millstone E., (2001) «'mad cow disease' 1980s - 2000: how reassurances undermined precaution», dans Late lessons from early warnings : the precautionary principle 1896 2000, EEA, Copenhagen, pp. 157 - 167.

Zangerl A. R., McKenna D., Wraight C. L., Carroll M., Ficarello P., Warner R. and Berenbaum M. R. (2001), «Effects of exposure to event 176 Bacillus thuringiensis corn pollen on monarch and black swallowtail caterpillars under field conditions », Proc. Natl. Acad. Sci., Vol. 98, pp. 1190811912.

\section{Annexe 1 - Les principaux arguments qui apparaissent dans le débat public sur les OGM en termes de risques}

\begin{tabular}{|c|c|c|}
\hline & Type de dommage & $\begin{array}{l}\text { Méthode d'identification/ } \\
\text { validation }^{71}\end{array}$ \\
\hline \multicolumn{3}{|l|}{ Consommateurs } \\
\hline $\begin{array}{l}\text { Contamination de denrées alimentaires } \\
\text { si une stricte séparation des filières } \\
\text { n'est pas mise en place }\end{array}$ & $\begin{array}{l}\text { Sanitaire, éthique, } \\
\text { économique }\end{array}$ & $\begin{array}{l}\text { Scientifiques } \\
\text { Autres: p.ex. analyse } \\
\text { des filières }\end{array}$ \\
\hline $\begin{array}{l}\text { Ne plus être en mesure de choisir } \\
\text { une alimentation exempte d'OGM } \\
\text { si un système d'étiquetage } \\
\text { n'est pas rendu obligatoire }\end{array}$ & $\begin{array}{l}\text { Sanitaire, éthique, } \\
\text { économique }\end{array}$ & $\begin{array}{l}\text { Scientifiques } \\
\text { Autres: p. ex. analyse } \\
\text { des filières } \\
\text { et des systèmes } \\
\text { de transformation, } \\
\text { conditionnement, etc. }\end{array}$ \\
\hline$\ldots$ & $\ldots$ & $\ldots$ \\
\hline \multicolumn{3}{|l|}{$\underline{\text { Paysans }}$} \\
\hline $\begin{array}{l}\text { Contamination de champs non OGM } \\
\text { par des champs voisins OGM }\end{array}$ & $\begin{array}{l}\text { Environnemental, } \\
\text { économique, éthique }\end{array}$ & Scientifiques \\
\hline $\begin{array}{l}\text { Risque pour la biodiversité } \\
\text { des plantes agricoles }\end{array}$ & $\begin{array}{l}\text { Environnemental, } \\
\text { économique, éthique }\end{array}$ & Scientifiques \\
\hline
\end{tabular}

Les méthodes d'identification et de validation des risques sont classées ici en deux catégories principales: d'une part les méthodes scientifiques et d'autre part les méthodes considérées comme non scientifiques par les instances de régulation et qui figurent sous le terme «autres» dans le tableau. L'établissement de ces deux catégories se justifie du fait qu'aux méthodes d'identification-validation scientifiques correspondent les risques reconnus, alors qu'aux « autres » méthodes correspondent les risques qui ne sont pas pris en compte par les instances de régulation, les risques non reconnus, autrement dit les menaces, selon la définition que nous en avons faite dans cet article. Enfin, dans la mesure du possible, nous avons tenté de préciser quelles sont les méthodes qui, bien que considérées comme non scientifiques, sont susceptible d'identifier et de valider les risques économiques, sociaux ou éthiques. 


\begin{tabular}{|c|c|c|}
\hline & Type de dommage & $\begin{array}{l}\text { Méthode d'identification/ } \\
\text { validation }\end{array}$ \\
\hline $\begin{array}{l}\text { Développement de résistance } \\
\text { chez les insectes (en ce qui concerne } \\
\text { les plantes Bt) pouvant réduire } \\
\text { l'efficacité de traitement } \\
\text { avec l'insecticide Bt, en particulier } \\
\text { sur les cultures biologiques où il est } \\
\text { l'un des seuls insecticides autorisés. }\end{array}$ & $\begin{array}{l}\text { Environnemental, } \\
\text { économique }\end{array}$ & Scientifiques \\
\hline $\begin{array}{l}\text { Risque lié à l'irréversibilité du choix } \\
\text { d'adopter des plantes transgéniques: } \\
\text { une fois que des OGM seront } \\
\text { disséminés dans l'environnement, } \\
\text { tout risque d'être contaminé } \\
\text { et il ne sera plus possible de revenir } \\
\text { en arrière. }\end{array}$ & Economique, éthique & Autres \\
\hline $\begin{array}{l}\text { En Suisse, risque de se retrouver } \\
\text { en porte-à-faux avec la politique } \\
\text { agricole qui va dans la direction } \\
\text { de la production intégrée } \\
\text { et de la production biologique }\end{array}$ & Politique, social & $\begin{array}{l}\text { Autres: p.ex. analyse } \\
\text { socio-politique, } \\
\text { analyse } \\
\text { de la compatibilité } \\
\text { entre la politique } \\
\text { agricole suisse } \\
\text { et le développement } \\
\text { de plantes } \\
\text { transgéniques }\end{array}$ \\
\hline $\begin{array}{l}\text { Risque de voir la production OGM } \\
\text { refusée par les consommateurs } \\
\text { (risque de ne pas trouver de marché) }\end{array}$ & Economique & $\begin{array}{l}\text { Autres: p. ex. étude } \\
\text { de marché }\end{array}$ \\
\hline $\begin{array}{l}\text { Dépendance accrue vis-à-vis } \\
\text { de l'agro-industrie; } \\
\text { ce risque est invoqué aussi bien par } \\
\text { des paysans en Suisse que } \\
\text { par des paysans dans des pays du Sud }\end{array}$ & Economique, social & $\begin{array}{l}\text { Autres: p. ex. analyse } \\
\text { socio-économique }\end{array}$ \\
\hline $\begin{array}{l}\text { Risque de faire «comme avec } \\
\text { la vache folle», à savoir de répondre } \\
\text { à des impératifs de rentabilité } \\
\text { économique afin de produire plus } \\
\text { et d'aboutir finalement } \\
\text { à une catastrophe économique, } \\
\text { sociale et sanitaire. }\end{array}$ & $\begin{array}{l}\text { Economique, social, } \\
\text { sanitaire }\end{array}$ & $\begin{array}{l}\text { Autres: p. ex. analyse } \\
\text { socio-économique }\end{array}$ \\
\hline$\ldots$ & $\ldots$ & $\ldots$ \\
\hline \multicolumn{3}{|l|}{$\underline{\text { Semencier }}$} \\
\hline $\begin{array}{l}\text { Risque de voir les paysans refuser } \\
\text { les semences transgéniques } \\
\text { (pas de marché) }\end{array}$ & Economique & $\begin{array}{l}\text { Autres: p. ex. étude } \\
\text { de marché }\end{array}$ \\
\hline
\end{tabular}




\begin{tabular}{|c|c|c|}
\hline & Type de dommage & $\begin{array}{l}\text { Méthode d'identification/ } \\
\text { validation }\end{array}$ \\
\hline $\begin{array}{l}\text { Risque quant à la responsabilité } \\
\text { du semencier au cas où il y aurait } \\
\text { des dommages }\end{array}$ & Juridique & $\begin{array}{l}\text { Autres: p. ex. } \\
\text { législation en vigueur }\end{array}$ \\
\hline$\ldots$ & $\ldots$ & $\ldots$ \\
\hline \multicolumn{3}{|l|}{ Mouvement altermondialiste } \\
\hline $\begin{array}{l}\text { Domination accrue } \\
\text { des multinationales }\end{array}$ & Social, économique & $\begin{array}{l}\text { Autres: analyse } \\
\text { socio-économique }\end{array}$ \\
\hline $\begin{array}{l}\text { Accroissement de la pauvreté } \\
\text { et des inégalités Nord-Sud }\end{array}$ & $\begin{array}{l}\text { Social, éthique, } \\
\text { économique }\end{array}$ & $\begin{array}{l}\text { Autres: p. ex. analyse } \\
\text { socio-économique }\end{array}$ \\
\hline $\begin{array}{l}\text { Pillage des ressources génétiques } \\
\text { de pays du Sud grâce au système } \\
\text { des brevets }\end{array}$ & $\begin{array}{l}\text { Ethique, social, } \\
\text { économique }\end{array}$ & $\begin{array}{l}\text { Autres: p. ex. analyse } \\
\text { de la législation } \\
\text { sur les brevets, } \\
\text { de l'accord ADPICs }\end{array}$ \\
\hline $\begin{array}{l}\text { Risque pour la sécurité alimentaire } \\
\text { en particulier dans les pays } \\
\text { en développement (brevets) }\end{array}$ & Social & $\begin{array}{l}\text { Autres: p. ex. étude } \\
\text { de la situation } \\
\text { alimentaire, } \\
\text { socio-économique } \\
\text { et légale }\end{array}$ \\
\hline $\begin{array}{l}\text { Risque pour la liberté de choix } \\
\text { et pour l'autodétermination } \\
\text { alimentaire }\end{array}$ & Ethique & $\begin{array}{l}\text { Autres: p. ex. étude } \\
\text { de la situation } \\
\text { alimentaire, socio- } \\
\text { économique et légale }\end{array}$ \\
\hline $\begin{array}{l}\text { Risque d'extension de la loi } \\
\text { du marché et du profit à un domaine } \\
\text { vital tel que l'alimentation }\end{array}$ & Social, économique & $\begin{array}{l}\text { Autres: p. ex. étude } \\
\text { de la situation } \\
\text { alimentaire, socio- } \\
\text { économique et légale }\end{array}$ \\
\hline $\begin{array}{l}\text { Risque pour la mise en place } \\
\text { d'un ordre mondial respectant } \\
\text { à la fois l'homme et l'environnement }\end{array}$ & Ethique, social & $\begin{array}{l}\text { Autres: } \\
\text { étude socio-politique } \\
\text { et éthique }\end{array}$ \\
\hline$\ldots$ & $\ldots$ & $\ldots$ \\
\hline \multicolumn{3}{|l|}{ Ecologistes } \\
\hline $\begin{array}{l}\text { Dissémination de gènes marqueurs } \\
\text { de résistance aux antibiotiques } \\
\text { présents dans les plantes } \\
\text { transgéniques et donc risque } \\
\text { d'accroissement des résistances } \\
\text { chez des bactéries pathogènes } \\
\text { pour l'homme. }\end{array}$ & Sanitaire & Scientifiques \\
\hline $\begin{array}{l}\text { Dissémination de transgènes } \\
\text { dans l'environnement }\end{array}$ & Environnemental & Scientifiques \\
\hline
\end{tabular}




\begin{tabular}{|c|c|c|}
\hline & Type de dommage & $\begin{array}{l}\text { Méthode d'identification/ } \\
\text { validation }\end{array}$ \\
\hline Risque pour la biodiversité & $\begin{array}{l}\text { Environnemental, } \\
\text { éthique }\end{array}$ & Scientifiques \\
\hline $\begin{array}{l}\text { Risques environnementaux } \\
\text { irréversibles et imprévisibles } \\
\text { surtout à long terme }\end{array}$ & Environnemental & Scientifiques \\
\hline $\begin{array}{l}\text { Risques alimentaires liés } \\
\text { à l'instabilité génétique des OGM }\end{array}$ & Sanitaire (alimentaire) & Scientifiques \\
\hline Risques alimentaires imprévisibles & Sanitaire (alimentaire) & Scientifique \\
\hline $\begin{array}{l}\text { Dissémination de gènes de résistance } \\
\text { aux herbicides et aux insecticides }\end{array}$ & Environnemental & Scientifiques \\
\hline $\begin{array}{l}\text { Risque de déséquilibrer la nature } \\
\text { en y introduisant des plantes } \\
\text { «Frankenstein» }\end{array}$ & Environnemental & Scientifiques \\
\hline $\begin{array}{l}\text { Risque de franchir un échelon } \\
\text { supplémentaire } \\
\text { dans l'instrumentalisation de la nature }\end{array}$ & Ethique & Autres \\
\hline $\begin{array}{l}\text { Risque pour «l'intégrité } \\
\text { de la créature», risque que } \\
\text { les organismes vivants soient réduits } \\
\text { à l'état de choses }\end{array}$ & Ethique & Autres \\
\hline$\ldots$ & $\ldots$ & $\ldots$ \\
\hline \multicolumn{3}{|l|}{ Croyants } \\
\hline Risques pour l'intégrité de la créature & Ethique & Autres \\
\hline $\begin{array}{l}\text { Risque de voir l'homme se substituer } \\
\text { à Dieu en «créant» de nouveau } \\
\text { organismes vivants et en modifiant } \\
\text { le cours de l'évolution }\end{array}$ & Ethique & Autres \\
\hline$\ldots$ & $\ldots$ & $\ldots$ \\
\hline \multicolumn{3}{|l|}{ Industrie agrochimique } \\
\hline $\begin{array}{l}\text { Pour les industries européennes, } \\
\text { la perte de compétitivité face } \\
\text { aux industries américaines } \\
\text { si le moratoire persiste } \\
\text { et si les procédures d'autorisation } \\
\text { deviennent encore plus strictes }\end{array}$ & Economique & $\begin{array}{l}\text { Autres: p. ex. études } \\
\text { socio-économiques }\end{array}$ \\
\hline $\begin{array}{l}\text { Fuite des cerveaux vers les pays ayant } \\
\text { une législation favorisant la recherche } \\
\text { en biotechnologie végétale }\end{array}$ & Economique, social & $\begin{array}{l}\text { Autres: p. ex. études } \\
\text { socio-économiques }\end{array}$ \\
\hline $\begin{array}{l}\text { Risque quant à la responsabilité } \\
\text { de l'entreprise au cas où il y aurait } \\
\text { des dommages }\end{array}$ & Juridique & $\begin{array}{l}\text { Autres: p. ex. étude } \\
\text { de la législation } \\
\text { en vigueur }\end{array}$ \\
\hline
\end{tabular}




\section{Annexe 2 - Les différents instruments de régulation}

\begin{tabular}{|c|c|c|c|}
\hline & Accords & Objet valorisé & $\begin{array}{l}\text { Méthode d'identification / } \\
\text { validation reconnue }\end{array}$ \\
\hline $\bar{\sigma}$ & $\begin{array}{l}\text { Protocole de Cartagena } \\
\text { sur les risques } \\
\text { biotechnologiques relatif } \\
\text { à la convention sur } \\
\text { la diversité biologique }\end{array}$ & $\begin{array}{l}\text { Conservation et utilisation } \\
\text { durable de la diversité } \\
\text { biologique (art. 1) } \\
\text { Santé humaine (art. 1) } \\
\text { Incidences socio-économiques } \\
\text { de l'impact des organismes } \\
\text { vivants modifiés sur } \\
\text { la conservation et l'utilisation } \\
\text { durable de la diversité } \\
\text { biologique, eu égard } \\
\text { à la valeur de la diversité } \\
\text { biologique pour les } \\
\text { communautés autochtones } \\
\text { et locales, en particulier } \\
\text { (art. 26). }\end{array}$ & $\begin{array}{l}\text { Méthodes scientifiques } \\
\text { éprouvées (art. 15.1; } \\
\text { Annexe III, al. 3) }\end{array}$ \\
\hline 10 & $\begin{array}{l}\text { Accord sur l'application } \\
\text { des mesures sanitaires } \\
\text { et phytosanitaires (SPS) } \\
\text { de l'OMC }\end{array}$ & $\begin{array}{l}\text { Protéger la santé et la vie } \\
\text { des personnes et des animaux } \\
\text { ou préserver les végétaux } \\
\text { (art. 2.1) }\end{array}$ & $\begin{array}{l}\text { Principes scientifiques } \\
\text { (art. 2.2) }\end{array}$ \\
\hline 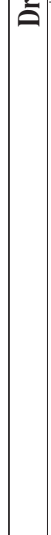 & Codex alimentarius & $\begin{array}{l}\text { Protecting the health } \\
\text { of the consumers and ensuring } \\
\text { fair practices in the food trade } \\
\text { (art. 1.a, Statutes of the Codex } \\
\text { Alimentarius Commission) }\end{array}$ & $\begin{array}{l}\text { The principle of sound } \\
\text { scientific analysis } \\
\text { and evidence (al. 1, } \\
\text { Statement of the } \\
\text { Principle Concerning } \\
\text { the role of Science } \\
\text { in the Codex Decision- } \\
\text { Making Process } \\
\text { and the Extent to which } \\
\text { other factors are taken } \\
\text { into account, Appendix: } \\
\text { general decisions } \\
\text { of the Commission) } \\
\text { Other legitimate factors } \\
\text { (al. 2, idem) }\end{array}$ \\
\hline
\end{tabular}




\begin{tabular}{|c|c|c|c|}
\hline & Accords & Objet valorisé & $\begin{array}{l}\text { Méthode d'identification / } \\
\text { validation reconnue }\end{array}$ \\
\hline \multirow[t]{2}{*}{ 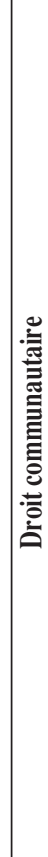 } & Directive 2001/18/CE & $\begin{array}{l}\text { La santé humaine } \\
\text { et l'environnement (art. 1) } \\
\text { Obligation d'étiquetage } \\
\text { des OGM en tant que produits } \\
\text { ou éléments de produits } \\
\text { (art. } 21 \text { ) } \\
\text { NB : l'objet valorisé à travers } \\
\text { cette disposition est la liberté } \\
\text { de choix des consommateurs } \\
\text { Principes éthiques } \\
\text { (Considérant 9). } \\
\text { NB : La directive prévoit } \\
\text { en outre la consultation } \\
\text { par la Commission de comités } \\
\text { d'éthique afin d'être } \\
\text { conseillée sur les implications } \\
\text { éthiques des biotechnologies. } \\
\text { (art. 29.2) }\end{array}$ & $\begin{array}{l}\text { Méthodologie commune } \\
\text { d'évaluation des risques } \\
\text { basée sur une } \\
\text { consultation scientifique } \\
\text { indépendante } \\
\text { (Considérant 20), } \\
\text { Méthode } \\
\text { scientifiquement fiable, } \\
\text { fondée sur les données } \\
\text { scientifiques et } \\
\text { techniques disponibles } \\
\text { (Annexe II, B) }\end{array}$ \\
\hline & $\begin{array}{l}\text { Règlement 258/97 } \\
\text { Règlement (CE) 1139/98 } \\
\text { Règlement (CE) 49/2000 } \\
\text { Règlement (CE) 50/2000 }\end{array}$ & $\begin{array}{l}\text { Etiquetage de denrées } \\
\text { alimentaires contenant } \\
\text { des OGM } \\
\text { NB : l'objet valorisé à travers } \\
\text { cette disposition est la liberté } \\
\text { de choix des consommateurs }\end{array}$ & \\
\hline
\end{tabular}




\begin{tabular}{|c|c|c|c|}
\hline & Accords & Objet valorisé & $\begin{array}{l}\text { Méthode d'identification } \\
\text { validation reconnue }\end{array}$ \\
\hline & $\begin{array}{l}\text { Constitution fédérale } \\
\text { (Suisse) }\end{array}$ & $\begin{array}{l}\text { L'être humain et son } \\
\text { environnement naturel } \\
\text { (art. 74.1) (art.120.1) } \\
\text { L'intégrité des organismes } \\
\text { vivants et la sécurité de l'être } \\
\text { humain, de l'animal } \\
\text { et de l'environnement } \\
\text { et la diversité génétique } \\
\text { des espèces animales } \\
\text { et végétales. (art.120.2) }\end{array}$ & \\
\hline 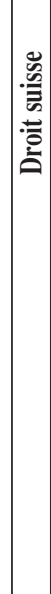 & $\begin{array}{l}\text { Loi fédérale sur } \\
\text { l'application du génie } \\
\text { génétique au domaine } \\
\text { non humain (Loi génie } \\
\text { génétique, LGG) }\end{array}$ & $\begin{array}{l}\text { L'être humain, les animaux } \\
\text { et l'environnement (art. 1.1.a) } \\
\text { La santé et la sécurité } \\
\text { de l'être humain, des animaux } \\
\text { et de l'environnement } \\
\text { (art. 1.2. a) (art. 6) } \\
\text { La diversité biologique } \\
\text { et la fertilité du sol } \\
\text { (art. 1.2.b) (art. 6) } \\
\text { L'intégrité des organismes } \\
\text { vivants (art.1.2.c) (art. 8) } \\
\text { Le libre choix } \\
\text { des consommateurs } \\
\text { (art.1.2.d) (art. 7) } \\
\text { Production exempte } \\
\text { d'organismes génétiquement } \\
\text { modifiés (art. 7) }\end{array}$ & $\begin{array}{l}\text { «d'après les } \\
\text { connaissances } \\
\text { scientifiques les plus } \\
\text { récentes» (art. 6.2.d) }\end{array}$ \\
\hline
\end{tabular}

